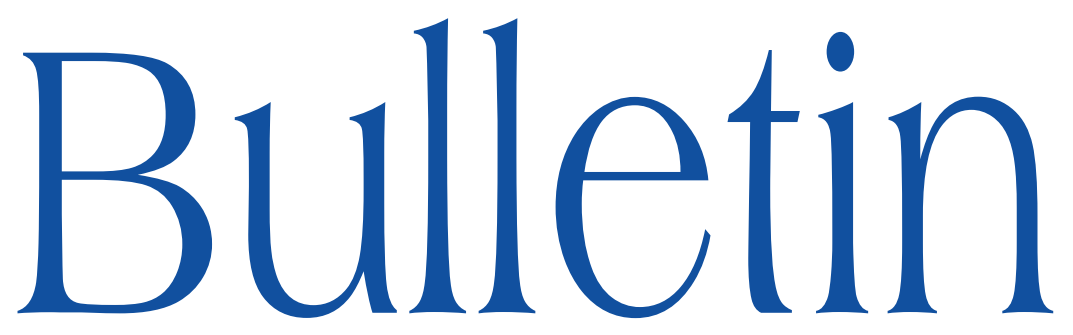

de la SOCIÉTÉ MATHÉMATIQUE DE FRANCE

\title{
INTRINSIC PSEUDO-VOLUME FORMS FOR LOGARITHMIC PAIRS
}

Thomas Dedieu

\section{Tome 138}

Fascicule 4 


\title{
INTRINSIC PSEUDO-VOLUME FORMS FOR LOGARITHMIC PAIRS
}

\author{
BY ThOMAS DEDIEU
}

\begin{abstract}
We study an adaptation to the logarithmic case of the KobayashiEisenman pseudo-volume form, or rather an adaptation of its variant defined by Claire Voisin, for which she replaces holomorphic maps by holomorphic $K$-correspondences. We define an intrinsic logarithmic pseudo-volume form $\Phi_{X, D}$ for every pair $(X, D)$ consisting of a complex manifold $X$ and a normal crossing Weil divisor $D$ on $X$, the positive part of which is reduced. We then prove that $\Phi_{X, D}$ is generically nondegenerate when $X$ is projective and $K_{X}+D$ is ample. This result is analogous to the classical Kobayashi-Ochiai theorem. We also show the vanishing of $\Phi_{X, D}$ for a large class of log- $K$-trivial pairs, which is an important step in the direction of the Kobayashi conjecture about infinitesimal measure hyperbolicity in the logarithmic case.
\end{abstract}

\section{Résumé (Pseudo-formes volumes intrinsèques pour les paires logarithmiques)}

Nous étudions une adaptation au cas logarithmique de la pseudo-forme volume de Kobayashi-Eisenman, ou plutôt une adaptation de sa variante définie par Claire Voisin, pour laquelle elle remplace les applications holomorphes par des $K$-correspondances holomorphes. Nous définissons une pseudo-forme volume logarithmique intrinsèque $\Phi_{X, D}$ pour toute paire $(X, D)$ constituée d'une variété complexe $X$ et d'un diviseur de Weil à croisements normaux $D$ sur $X$, dont la partie positive est réduite. Nous prouvons que $\Phi_{X, D}$ est génériquement non dégénérée quand $X$ est projective et $K_{X}+$ $D$ est ample. Ce résultat est analogue au théorème de Kobayashi-Ochiai classique.

Texte reçu le 5 mai 2008, révisé le 11 mai 2009, accepté le 12 mai 2009

Thоmas Dedieu, Université Paul Sabatier, Institut de Mathématiques de Toulouse, 118 route de Narbonne, F-31062 Toulouse Cedex 9, France - E-mail : dedieu@math.jussieu.fr

Url : http://www.institut.math.jussieu.fr/ dedieu/

2000 Mathematics Subject Classification. - 32H99, 32Q45, 14J32.

Key words and phrases. - log- $K$-correspondence; Kobayashi-Eisenman pseudo-volume form; logarithmic pair. 
Nous montrons aussi l'annulation de $\Phi_{X, D}$ pour une grande classe de paires log- $K$ triviales, ce qui est une étape importante en direction de la conjecture de Kobayashi sur l'hyperbolicité au sens de la mesure infinitésimale dans le cas logarithmique.

\section{Introduction}

In the standard non logarithmic case, Kobayashi and Eisenman have defined an intrinsic pseudo-volume form $\Psi_{X}$ on every complex manifold $X$ ([13]). The definition involves all holomorphic maps from the unit polydisk $\mathbf{D}^{n} \subset \mathbf{C}^{n}$ to $X . \Psi_{X}$ coincides with the Poincaré hyperbolic volume form on $X$ when $X$ is a quotient (by a group acting freely and properly discontinuously) of the unit polydisk $\mathbf{D}^{n}$. In fact, if $X$ is a smooth curve of genus $g$, then we have the following dichotomy as a consequence of the Klein-Poincaré uniformization theorem: if $g=0$ or $g=1$, then the universal covering of $X$ is $\mathbf{P}^{1}$ or $\mathbf{C}$, and $\Psi_{X}$ vanishes; if $g \geqslant 2$, then the universal covering of $X$ is the unit disk $\mathbf{D}$, and $\Psi_{X}$ is induced by the Poincaré volume form on $\mathbf{D}$. For an $n$-dimensional manifold $X$, one expects the situation to follow the same outline. This is in part proved by the Kobayashi-Ochiai theorem ([15]), which states that if $X$ is of general type, then $\Psi_{X}$ is non degenerate outside a proper closed algebraic subset of $X$. A variety $X$ such that $\Psi_{X}>0$ almost everywhere is said to be infinitesimal measure hyperbolic. On the other hand, Kobayashi conjectured that if $X$ is not of general type, then $\Psi_{X}$ vanishes on a Zariski open subset of $X$. The Kobayashi conjecture is proved in the 2-dimensional case for algebraic varieties, using the classification of surfaces (see [9]): Green and Griffiths show that $\Psi_{X}=0$ on a dense Zariski open set when $X$ is covered by abelian varieties, and use the fact that algebraic $K 3$ surfaces are swept out by elliptic curves.

It is indeed an important step towards the Kobayashi conjecture to show that if $X$ is a Calabi-Yau variety, then $\Psi_{X}$ vanishes generically. In [24], Claire Voisin defines a new intrinsic pseudo-volume form $\Phi_{X, a n}$, which is a variant of $\Psi_{X}$, and for which she is able to show that a very wide range of Calabi-Yau varieties satisfy the Kobayashi conjecture (in fact, she shows that the pseudovolume form $\Phi_{X, a n}$ vanishes on these varieties). She also proves a theorem relative to $\Phi_{X, a n}$, which is exactly analogous to the Kobayashi-Ochiai theorem. The definition of $\Phi_{X, a n}$ is obtained from the definition of $\Psi_{X}$ by replacing the holomorphic maps from $\mathbf{D}^{n}$ to $X$ by $K$-correspondences. A $K$-correspondence between two complex manifolds $X$ and $Y$ of the same dimension is a closed analytic subset $\Sigma \subset X \times Y$ satisfying the following properties:

(i) the projections $\Sigma \rightarrow X$ and $\Sigma \rightarrow Y$ are generically of maximal rank on each irreducible component of $\Sigma$, 
(ii) the first projection $\Sigma \rightarrow X$ is proper,

(iii) for every desingularization $\tau: \widetilde{\Sigma} \rightarrow \Sigma$, letting $f:=\operatorname{pr}_{1} \circ \tau$ and $g:=\operatorname{pr}_{2} \circ \tau$, one has the inequality $R_{f} \leqslant R_{g}$ between the ramification divisors of $f$ and $g$.

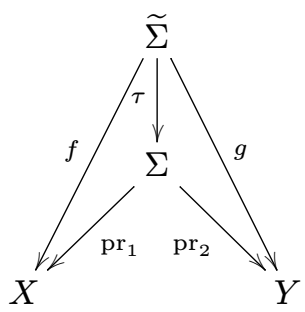

A $K$-correspondence $\Sigma$ has to be seen as the graph of a multivalued map between $X$ and $Y$. The last condition (iii) ensures the existence of a generalized Jacobian map $\left(J_{\widetilde{\Sigma}}\right)^{T}: g^{*} K_{Y} \rightarrow f^{*} K_{X}$. This definition of a $K$-correspondence, which was introduced in [24], derives from the notion of $K$-equivalence, for which both projections $\Sigma \rightarrow X$ and $\Sigma \rightarrow Y$ are birational.

It is nowadays understood that for certain problems, it is more relevant to consider logarithmic pairs $(X, D)$ rather than simply considering varieties. In this situation, one replaces the canonical bundle $K_{X}$ of $X$ by the log-canonical bundle $K_{X}(D)$. A first very classical example of this is given by the study of open varieties. If $U$ is a complex manifold, such that there exist a compact variety $X$ and a normal crossing divisor $D \subset X$, such that $U=X \backslash D$, then the study of the pair $(X, D)$ provides a lot of information about $U$. For example, the Betti cohomology with complex coefficients of $U$ can be computed as the hypercohomology of the logarithmic de Rham complex $\Omega_{X}^{\bullet}(\log D)$, see e.g. [22]. It has also been made clear, that the minimal model program has to be worked out for pairs, rather than simply for varieties. But the best clue, showing that it is indeed necessary to define an intrinsic pseudo-volume form for logarithmic pairs (analogous to $\Psi_{X}$ ), is perhaps the following.

In [3], Campana shows that to decompose a compact Kähler variety into components of special and hyperbolic types, one necessarily has to consider fibrations with orbifold bases. By definition, a complex manifold $X$ is of special type if there does not exist any non trivial meromorphic fibration $X \rightarrow Y$ with orbifold base of general type. Fano and $K$-trivial manifolds are special, but for every $n>0$ and $\kappa \in\{-\infty, 0,1, \ldots, n-1\}$, there exist $n$-dimensional manifolds $X$ with $\kappa(X)=\kappa$ that are special. If $Y$ is a complex manifold, an orbifold structure on $Y$ is the data of a $\mathbf{Q}$-divisor $\Delta=\sum_{j} a_{j} D_{j}$, where $0<a_{j} \leqslant 1$, $a_{j} \in \mathbf{Q}$, and the $D_{j}$ are distinct irreducible divisors on $Y$. The canonical bundle of the orbifold $(Y / \Delta)$ is the Q-divisor $K_{Y}+\Delta$ on $Y$. If $X$ and $Y$ are smooth complex varieties, and if $f: X \rightarrow Y$ is a holomorphic fibration, then 
the orbifold base of $f$ is $(Y / \Delta(f))$, where

$$
\Delta(f):=\sum_{D \subset Y}\left(1-\frac{1}{m(f, D)}\right) \cdot D,
$$

$m(f, D)$ being the multiplicity of the fiber of $f$ above the generic point of $D$. Campana constructs for every variety $X$ (or rather for every orbifold $(X / D)$ ) a functorial fibration $c_{X}: X \rightarrow C(X)$, the core of $X$, which is characterized by the fact that the generic fibers are special, and that the orbifold base is hyperbolic. In addition, he conjectures that the Kobayashi pseudo-metric $d_{X}$ on $X$ (note that this is not the same as the Kobayashi-Eisenman pseudo-volume form) is the pull-back via $c_{X}$ of a pseudo-metric $\delta_{X}$ on the orbifold base of the core.

In this paper, we seek the definition of a pseudo-volume form $\Phi_{X, D}$ on a logarithmic pair $(X, D)$. Let $X$ be a complex manifold of dimension $n$, and $D$ be a normal crossing Weil divisor on $X$, the positive part of which is reduced (we say that $D$ is a normal crossing divisor if its support has normal crossings). Note that we do not require that $D$ has a non zero positive part.

THEOREM 1. - (i) There exists a logarithmic pseudo-volume form $\Phi_{X, D}$ on the pair $(X, D)$, i.e. a pseudo-metric on the line bundle $\bigwedge^{n} T_{X}(-D)$, satisfying the following functoriality property. Let $Y$ be a complex manifold, and $\nu: Y \rightarrow$ $X$ be a proper morphism with ramification divisor $R$, such that $\nu^{*} D-R$ is a normal crossing divisor, the positive part of which is reduced. Then we have

$$
\nu^{*} \Phi_{X, D}=\Phi_{Y, \nu^{*} D-R}
$$

(when $\nu$ is not proper, we only get the inequality $\nu^{*} \Phi_{X, D} \leqslant \Phi_{Y, \nu^{*} D-R}$ ).

(ii) Let $D$ and $D^{\prime}$ be two normal crossing Weil divisors on $X$, the respective positive part of which are reduced. If $D \leqslant D^{\prime}$, then $\Phi_{X, D} \leqslant \Phi_{X, D^{\prime}}$.

(iii) If $D=0$, then $\Phi_{X, 0}=\Phi_{X, a n}$.

This is obtained by following the definition of $\Phi_{X, a n}$ in [24]. One replaces the holomorphic maps between $\mathbf{D}^{n}$ and $X$ in the definition of $\Psi_{X}$ by $\log -K$ correspondences between $\left(\mathbf{D}^{n}, \Delta_{k}\right)$ and $(X, D)$, where $\Delta_{k}$ is the divisor given in $\mathbf{D}^{n}$ by the equation $z_{n-k+1} \cdots z_{n}=0$. They are closed analytic subsets $\Sigma \subset \mathbf{D}^{n} \times X$, satisfying the following three properties : (i) the projections to $X$ and $Y$ are generically of maximal rank on each irreducible component of $\Sigma$, (ii) the first projection $\Sigma \rightarrow \mathbf{D}^{n}$ is proper, and (iii) with the same notations as in (1) above $\left(\tau: \widetilde{\Sigma} \rightarrow \Sigma\right.$ is a desingularization, $f=\operatorname{pr}_{1} \circ \tau$, and $\left.g=\operatorname{pr}_{2} \circ \tau\right)$

$$
R_{f}-f^{*} \Delta_{k} \leqslant R_{g}-g^{*} D .
$$

The ramification divisor $R_{f}$ (resp. $R_{g}$ ) is the zero divisor of the section of $K_{\widetilde{\Sigma}} \otimes\left(f^{*} K_{\mathbf{D}^{n}}\right)^{-1}$ (resp. $\left.K_{\widetilde{\Sigma}} \otimes\left(g^{*} K_{X}\right)^{-1}\right)$ given by the Jacobian map of $f$ (resp. 
$g$ ). Condition (iii) ensures the existence of a generalized Jacobian morphism $\left(J_{\widetilde{\Sigma}}\right)^{T}: g^{*} K_{X}(D) \rightarrow f^{*} K_{\mathbf{D}^{n}}\left(\Delta_{k}\right)$.

In [24], Claire Voisin uses local $K$-isocorrespondences to transport the Poincaré volume form of $\mathbf{D}^{n}$ to small open sets on $X$. Here we use local $\log -K$-isocorrespondences to transport the logarithmic Poincaré volume form of $\left(\mathbf{D}^{n}, \Delta_{k}\right)$. This is done in Section 2. Log- $K$-correspondences are defined and studied in Section 1.

Section 3 is devoted to the proof of the following result, which generalizes the Kobayashi-Ochiai theorem to our case.

Theorem 2. - Let $(X, D)$ be a pair consisting of a projective $n$-dimensional complex manifold $X$ and a normal crossing Weil divisor $D$ on $X$, the effective part of which is reduced and has global normal crossings. If $K_{X}+D$ is ample, then $\Phi_{X, D}>0$ (instead of $\Psi_{X}$, and indeed another motivation for our construction) away from a proper closed algebraic subset of $X$.

As in [24], this is proved by a standard curvature argument, namely an adaptation to our case of the Ahlfors-Schwarz lemma, which is more or less an incarnation of the maximum principle (see e.g. [6] or [23]). We also use a result of Carlson and Griffiths, about the existence of metrics with negative Ricci curvature on the complement of hypersurfaces of projective algebraic manifolds, enjoying some further properties of Kähler-Einstein type (see [4] and [11]).

Let $X$ and $Y$ be two projective complex manifolds of the same dimension, and assume that $X$ is of general type. Let $\nu: Y \rightarrow X$ be a dominant morphism. Using the volume decreasing property $\nu^{*} \Psi_{X} \leqslant \Psi_{Y}$, it is well known how to obtain an upper bound on $\operatorname{deg} \nu$ from the classical Kobayashi-Ochiai theorem. However, when $X$ is not of general type, then we cannot say much about the degree of $\nu$.

One of the major benefits we get by considering $\Phi_{X, D}$ instead of $\Psi_{X}$, is that we get much more precise decreasing volume properties ( $c f$. theorem 1 and Section 2.3). Indeed, by formula (2), where $D=0$, the standard volume decreasing inequality is even replaced by an equality for proper morphisms, the ramification divisors of which have normal crossings. Taking the ramification of a morphism $\nu: Y \rightarrow X$ into consideration gives a greater accuracy in the comparison between the intrinsic (logarithmic) pseudo-volume forms on $X$ and $Y$ respectively.

Combining this with Theorem 2, we obtain a way to control the degree of a dominant morphism $\nu: Y \rightarrow X$ even if $X$ is not of general type ( $X$ and $Y$ are again of the same dimension). Assume there exists a normal crossing Weil divisor $D$ on $X$, with positive part reduced and with global normal crossings, and such that $K_{X}+D$ is ample. One decomposes the ramification divisor $R$ 
of $\nu$ into $R=\nu^{*} D+E$, where $E$ is a Weil divisor of $Y$, which has a non zero negative part as soon as $D$ has a non zero positive part. Assume that $E$ has normal crossings, and that its negative part is reduced. Then, by integrating on $Y$ the decreasing volume inequality

$$
\nu^{*} \Phi_{X, D} \leqslant \Phi_{Y,-E},
$$

which is an equality if $\nu$ is proper, one gets the inequality

$$
\left(\int_{X} \Phi_{X, D}\right)(\operatorname{deg} \nu) \leqslant \int_{Y} \Phi_{Y,-E} .
$$

The pseudo-volume forms $\Phi_{X, D}$ and $\Phi_{Y,-E}$ are allowed to have poles along the positive part of $D$, and the negative part of $E$ respectively. They are however integrable at the neighbourhood of these poles, as in the same way the Poincaré volume form on the punctured disk $\mathbf{D} \backslash\{0\}$ is integrable at the neighbourhood of 0 . On the other hand, we have $\int_{X} \Phi_{X, D}>0$ by theorem 2, so inequality (4) yields an upper bound on $\operatorname{deg} \nu$.

Eventually, we prove our main result in Section 4, that for many $\log -K$ trivial pairs, the pseudo-volume form $\Phi_{X, D}$ vanishes. This, of course, has to be seen as a step in the direction of the logarithmic version of the Kobayashi conjecture. This is stated as follows.

TheOREM 3. - Let $Y$ be a smooth rationally connected variety, and $(X, D)$ be a pair such that $X \subset Y$ is a smooth hypersurface, $D$ is reduced and has normal crossings, and $D=X \cap X^{\prime}$, where $X^{\prime} \subset Y$ is a reduced hypersurface such that

$$
X+X^{\prime} \in\left|-K_{Y}\right| .
$$

Then the pseudo-volume form $\Phi_{X, D}$ vanishes.

Note that by adjunction, the variety $\bar{X}:=X \cup X^{\prime}$ has trivial canonical bundle. Using the equality of line bundles $K_{X}(D)=\left.\left(K_{\bar{X}}\right)\right|_{X}$, we see that the hypotheses impose that $K_{X}(D)$ is trivial. As in [24], the key point in the proof is the production of $\log -K$-autocorrespondences of the pair $(X, D)$, i.e. correspondences $\Sigma \subset X \times X$ satisfying the equality $R_{f}-f^{*} D=R_{g}-g^{*} D$, and with the additional dilating property

$$
f^{*} \eta_{X}=\lambda g^{*} \eta_{X}
$$

where $|\lambda| \neq 1$, and $\eta_{X}$ is a generator of $\mathrm{H}^{0}\left(X, K_{X}(D)\right)$. This construction is in some way analogous to the definition of the multiplication by a complex number on an elliptic curve, realized as a cubic plane curve. Another important feature in the proof is the use of pull-backs on differential forms induced by correspondences, following original ideas of Mumford ([18], see also Chapter 22 in [22]). One has to be slightly more careful with them than with pull-backs on cohomology, which are used in [24].

TOME $138-2010-\mathrm{N}^{\circ} 4$ 
Acknowledgements. - I wish to thank Claire Voisin for introducing me to this subject, and for suggesting this work to me. She helped me to overcome the traps and difficulties that appeared during its preparation, and answered all my questions with great clarity.

\section{Log- $K$-correspondences}

1.1. Definition and basic properties. - In this section, we define and study the notion of log- $K$-correspondence. This will be used in the next section to define and study properly our variant of the Kobayashi-Eisenman pseudo-volume form.

Definition 1.1. - Let $(X, D)$ and $\left(Y, D^{\prime}\right)$ be pairs of the same dimension, i.e. $X$ and $Y$ are complex manifolds of dimension $n$, and $D$ and $D^{\prime}$ are (not necessarily effective) Weil divisors of $X$ and $Y$ respectively. A log- $K$ correspondence from $(X, D)$ to $\left(Y, D^{\prime}\right)$ is a reduced $n$-dimensional closed analytic subset $\Sigma \subset X \times Y$, satisfying the three following properties.

(i) The projections to $X$ and $Y$ are generically of maximal rank on each irreducible component of $\Sigma$.

(ii) The first projection $\Sigma \rightarrow X$ is proper.

(iii) Let $\tau: \widetilde{\Sigma} \rightarrow \Sigma$ be a desingularization, $f=\operatorname{pr}_{1} \circ \tau: \widetilde{\Sigma} \rightarrow X$, and $g=\operatorname{pr}_{2} \circ \tau: \widetilde{\Sigma} \rightarrow Y$. The ramification divisors $R_{f}$ and $R_{g}$ (of $f$ and $g$ respectively) satisfy the inequality

$$
R_{f}-f^{*} D \leqslant R_{g}-g^{*} D^{\prime}
$$

The above notations are summed up in the following commutative diagram. They will be used very often without further notice in the end of this text.

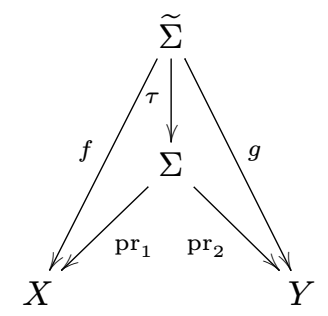

Note that if condition (iii) is true for one desingularization of $\Sigma$, then it is true for all desingularizations. Let us now explain the meaning of this last condition. The two Jacobian maps $\bigwedge^{n} d f$ and $\bigwedge^{n} d g$ (or rather their transpose) give isomorphisms of line bundles on $\widetilde{\Sigma}$

$$
f^{*}\left(K_{X}(D)\right) \cong K_{\widetilde{\Sigma}}\left(-R_{f}+f^{*} D\right) \quad \text { and } \quad g^{*}\left(K_{Y}\left(D^{\prime}\right)\right) \cong K_{\widetilde{\Sigma}}\left(-R_{g}+g^{*} D^{\prime}\right)
$$


So condition (iii) ensures the existence of a holomorphic (rather than just meromorphic) map

$$
\left(J_{\widetilde{\Sigma}}\right)^{T}: g^{*}\left(K_{Y}\left(D^{\prime}\right)\right) \rightarrow f^{*}\left(K_{X}(D)\right),
$$

given by the transpose of the holomorphic map

$$
J_{\widetilde{\Sigma}}:=\bigwedge^{n} d g \circ\left(\bigwedge^{n} d f\right)^{-1}: f^{*}\left(\bigwedge^{n} T_{X}(-D)\right) \rightarrow g^{*}\left(\bigwedge^{n} T_{Y}\left(-D^{\prime}\right)\right),
$$

which we call the generalized logarithmic Jacobian map. When $D=0$ and $D^{\prime}=0$, the notion of $\log -K$-correspondence coincides with the notion of $K$ correspondence introduced in [24].

It is also important to note the following inequality of divisors on $\widetilde{\Sigma}$. We write $D=D_{1}-D_{2}$, with both $D_{1}$ and $D_{2}$ non negative, and similarly $D^{\prime}=D_{1}^{\prime}-D_{2}^{\prime}$. If $D_{1}$ is reduced, then the negative part of $R_{f}-f^{*} D$ is the sum of the reduced divisor $\left(f^{*} D_{1}\right)_{\text {red }}$ and possibly of some $f$-exceptional components contained in $f^{*} D_{1}$. We write this $f$-exceptional sum $E_{1}$. In the same way, if $D_{1}^{\prime}$ is reduced, the negative part of $R_{g}-g^{*} D^{\prime}$ writes $\left(g^{*} D_{1}^{\prime}\right)_{\text {red }}+E_{1}^{\prime}$, where $E_{1}^{\prime}$ is a sum of $g$ exceptional components contained in $g^{*} D_{1}^{\prime}$. So if both $D_{1}$ and $D_{1}^{\prime}$ are reduced, then condition (iii) implies the inequality

$$
\left(f^{*} D_{1}\right)_{\mathrm{red}}+E_{1} \geqslant\left(g^{*} D_{1}^{\prime}\right)_{\mathrm{red}}+E_{1}^{\prime} .
$$

In particular, if $D^{\prime}$ has a positive part (i.e. if $D_{1}^{\prime}$ does not vanish), then $D$ necessarily has a positive part as well.

We shall now describe some enlightening examples.

ExAmPLe 1.2. - Let $X$ and $Y$ be complex manifolds of dimension $n$, and $D \subset$ $X$ be an effective divisor. A morphism $\varphi: X \rightarrow Y$ such that the ramification divisor $R_{\varphi}$ contains $D$ (with multiplicities) yields a morphism of line bundles

$$
\varphi^{*} K_{Y} \rightarrow K_{X}(-D) \text {. }
$$

The graph $\Gamma_{\varphi} \subset X \times Y$ is isomorphic to $X$. It satisfies properties (i) and (ii) of definition 1.1, and with the notations of (5), one has $R_{g}-R_{f}=R_{\varphi} \geqslant f^{*} D$. So $\Gamma_{\varphi}$ is a (smooth) $\log -K$-correspondence between $(X,-D)$ and $(Y, 0)$, and the generalized logarithmic Jacobian map

$$
g^{*} K_{Y} \rightarrow f^{*}\left(K_{X}(-D)\right)
$$

identifies to (6).

EXAMPLE 1.3. - Let $(X, D)$ and $\left(X^{\prime}, D^{\prime}\right)$ be smooth logarithmic pairs, where $X$ and $X^{\prime}$ are complex manifolds of the same dimension, and $D \subset X$ and $D^{\prime} \subset X^{\prime}$ are effective divisors. One also usually assumes $D$ and $D^{\prime}$ to be normal crossing divisors, but this is not necessary for this example. A morphism of pairs $\varphi:(X, D) \rightarrow\left(X^{\prime}, D^{\prime}\right)$ is a morphism of complex manifolds $X \rightarrow X^{\prime}$, such that the ramification divisor $R_{\varphi}$ contains $\varphi^{*} D^{\prime}-D$. In other words, we require that there exists an effective divisor $R \subset X$, such that $K_{X}+D=$ 
$\varphi^{*}\left(K_{X^{\prime}}+D^{\prime}\right)+R$ as divisors on $X$. Such a morphism yields a morphism of line bundles on $X$

$$
\varphi^{*}\left(K_{X^{\prime}}\left(D^{\prime}\right)\right) \rightarrow K_{X}(D) .
$$

Again, the graph $\Gamma_{\varphi} \subset X \times Y$ is isomorphic to $X$, and satisfies both properties (i) and (ii) of definition 1.1, and we have $R_{g}-R_{f}=R_{\varphi} \geqslant g^{*} D^{\prime}-f^{*} D$. So $\Gamma_{\varphi}$ is a log-K-correspondence between $(X, D)$ and $\left(X^{\prime}, D^{\prime}\right)$, and the generalized logarithmic Jacobian map

$$
g^{*}\left(K_{X^{\prime}}\left(D^{\prime}\right)\right) \rightarrow f^{*}\left(K_{X}(D)\right)
$$

identifies to $(7)$.

Finally, the following notion of log- $K$-isocorrespondence will be useful later.

Definition 1.4. - Let $(X, D)$ and $\left(Y, D^{\prime}\right)$ be logarithmic pairs of the same dimension $n$, and let $\Sigma \subset X \times Y$ be a reduced closed analytic subset, generically finite over $X$ and $Y$. We let $\tau: \widetilde{\Sigma} \rightarrow \Sigma$ be a desingularization, and use the notations (5). If both projections $\mathrm{pr}_{1}$ and $\mathrm{pr}_{2}$ are proper, and if

$$
R_{f}-f^{*} D=R_{g}-g^{*} D^{\prime},
$$

then $\Sigma$ is a log- $K$-isocorrespondence between $(X, D)$ and $\left(Y, D^{\prime}\right)$.

Note that under these hypotheses, $\Sigma$ is a $\log -K$-correspondence between $(X, D)$ and $\left(Y, D^{\prime}\right)$, and its transpose $\Sigma^{T} \subset Y \times X$ is a log- $K$-correspondence between $\left(Y, D^{\prime}\right)$ and $(X, D)$. The generalized logarithmic Jacobian map then induces an isomorphism of line bundles on $\widetilde{\Sigma}$

$$
g^{*}\left(K_{Y}\left(D^{\prime}\right)\right) \cong f^{*}\left(K_{X}(D)\right) .
$$

ExAmple 1.5. - We consider the unit disk $\mathbf{D}$, with maps $f: z \in \mathbf{D} \mapsto z^{p} \in \mathbf{D}$ and $g: z \in \mathbf{D} \mapsto z^{q} \in \mathbf{D}$, where $p$ and $q$ are two relatively prime integers. Then the diagram

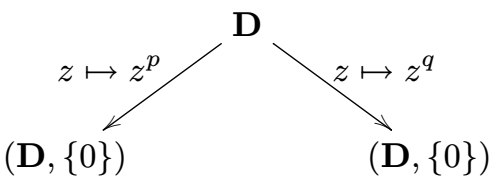

yields a log- $K$-autocorrespondence of the pair $(\mathbf{D},\{0\})$. Indeed, we have the equality of divisors on $\mathbf{D}$

$$
R_{f}-f^{*}\{0\}=(p-1)\{0\}-p\{0\}=-\{0\}=R_{g}-g^{*}\{0\} .
$$


1.2. Composition of log- $K$-correspondences. - We shall now study carefully the composition of two log- $K$-correspondences. This will allow us in the next section to prove some properties of volume decreasing type for our logarithmic pseudo-volume form.

We first need to define the weaker notion of 0 -correspondence, and to study the composition of two of them. Let $X$ and $Y$ be two $n$-dimensional complex manifolds.

Definition 1.6. - A 0-correspondence between $X$ and $Y$ is a reduced closed analytic subset $\Sigma \subset X \times Y$, which is generically finite over $X$ and $Y$, and such that the first projection $\Sigma \rightarrow X$ is proper.

In other words, $\Sigma \subset X \times Y$ has only to satisfy conditions (i) and (ii) of definition 1.1 to be a 0 -correspondence.

Let $Z$ be a third $n$-dimensional complex manifold. We denote by $p_{l q}$ the projection of $Z \times X \times Y$ to the $l$-th and $q$-th factors.

Proposition-Definition 1.7. - Let $\Sigma \subset X \times Y$ and $\Sigma^{\prime} \subset Z \times X$ be two 0 -correspondences. We define $\Sigma \circ \Sigma^{\prime} \subset Z \times Y$ as the union of the components of $p_{13}\left(p_{12}^{-1}\left(\Sigma^{\prime}\right) \cap p_{23}^{-1}(\Sigma)\right)$ on which the projections to $Z$ and $Y$ are generically of maximal rank. Then $\Sigma \circ \Sigma^{\prime} \subset Z \times Y$ is a 0 -correspondence.

Before stating the proof of this, let us see on a simple example why it may be necessary to remove certain irreducible components of $p_{13}\left(p_{12}^{-1}\left(\Sigma^{\prime}\right) \cap p_{23}^{-1}(\Sigma)\right)$. Assume for simplicity that $Z, X$ and $Y$ are surfaces. Suppose we are given two 0-correspondences $\Sigma \subset X \times Y$ and $\Sigma^{\prime} \subset Z \times X$, and that there exist two irreducible curves $C_{Z} \subset Z$ and $C_{Y} \subset Y$, and a point $x_{0} \in X$, such that $\Sigma^{\prime}$ contains $C_{Z} \times\left\{x_{0}\right\}$ and $\Sigma$ contains $\left\{x_{0}\right\} \times C_{Y}$. In other words, $\Sigma^{\prime}$ contains a contraction of the curve $C_{Z}$ to the point $x_{0}$, and $\Sigma$ contains a blow-up of the point $x_{0}$ onto the curve $C_{Y}$. Then $C_{Z} \times C_{Y}$ is an irreducible component of $p_{13}\left(p_{12}^{-1}\left(\Sigma^{\prime}\right) \cap p_{23}^{-1}(\Sigma)\right)$ of dimension 2 . It is obvious that this component does not satisfy condition (i) of definition 1.1. Note that it would correspond to a blow-up of every point of $C_{X}$ onto the curve $C_{Z}$.

Proof. - We have a natural identification between $p_{12}^{-1}\left(\Sigma^{\prime}\right) \cap p_{23}^{-1}(\Sigma)$ and $\Sigma^{\prime} \times_{X}$ $\Sigma$. The first projection $\Sigma^{\prime} \times_{X} \Sigma \rightarrow \Sigma^{\prime}$ is proper by the stability of properness under base change ( $\Sigma \rightarrow X$ is proper by the definition of a 0 -correspondence). Since the projection $\Sigma^{\prime} \rightarrow Z$ is proper as well, the composition $\Sigma^{\prime} \times_{X} \Sigma \rightarrow$ $\Sigma^{\prime} \rightarrow Z$ is proper. A fortiori $\Sigma \circ \Sigma^{\prime}$ satisfies condition (ii) of definition 1.1. Now condition (i) of definition 1.1 is clearly satisfied, and one sees that a component of $p_{13}\left(p_{12}^{-1}\left(\Sigma^{\prime}\right) \cap p_{23}^{-1}(\Sigma)\right)$ which is generically of maximal rank over both $Z$ and $Y$ is necessarily of dimension $n$.

We now specify definition 1.7 to the case of log- $K$-correspondences.

TOME $138-2010-\mathrm{N}^{\mathrm{O}} 4$ 
Proposition 1.8. - Let $D_{Z}$ and $D_{Y}$ be Weil divisors of $Z$ and $Y$ respectively, and $D_{X}$ and $D_{X}^{\prime}$ be Weil divisors of $X$. Assume $\Sigma^{\prime}$ is a log-K-correspondence between $\left(Z, D_{Z}\right)$ and $\left(X, D_{X}^{\prime}\right)$, and $\Sigma$ is a log-K-correspondence between $\left(X, D_{X}\right)$ and $\left(Y, D_{Y}\right)$. If $D_{X}^{\prime} \geqslant D_{X}$, then $\Sigma \circ \Sigma^{\prime}$ is a log-K-correspondence between $\left(Z, D_{Z}\right)$ and $\left(Y, D_{Y}\right)$.

We have to prove that the two generalized logarithmic Jacobian maps $J_{\widetilde{\Sigma}}^{T}$ : $g^{*}\left(K_{Y}\left(D_{Y}\right)\right) \rightarrow f^{*}\left(K_{X}\left(D_{X}\right)\right)$ and $J_{\widetilde{\Sigma}^{\prime}}^{T}: g^{\prime *}\left(K_{X}\left(D_{X}^{\prime}\right)\right) \rightarrow f^{\prime *}\left(K_{Z}\left(D_{Z}\right)\right)$ can be composed on $\widetilde{\Sigma}^{\prime} \times_{X} \widetilde{\Sigma}$, to obtain a generalized logarithmic Jacobian map for $\Sigma \circ \Sigma^{\prime}$. This composition is of course well defined, since we have a morphism $K_{X}\left(D_{X}\right) \rightarrow K_{X}\left(D_{X}^{\prime}\right)$ of line bundles on $X$, because of the inequality $D_{X} \leqslant$ $D_{X}^{\prime}$. We just have to show that all this lifts to a desingularization $\widetilde{\Sigma}^{\prime \prime} \rightarrow \Sigma \circ \Sigma^{\prime}$, and that the map we obtain in this way is actually the generalized logarithmic Jacobian map of $\Sigma \circ \Sigma^{\prime}$. This is a consequence of the following Lemma 1.9.

We need some further notations to state the lemma properly. We let $\tau^{\prime}$ : $\widetilde{\Sigma}^{\prime} \rightarrow \Sigma^{\prime}$ and $\tau: \widetilde{\Sigma} \rightarrow \Sigma$ be desingularizations of $\Sigma^{\prime}$ and $\Sigma$ respectively. We call $f^{\prime}$ and $g^{\prime}$ (resp. $f$ and $g$ ) the maps from $\widetilde{\Sigma}^{\prime}$ (resp. $\widetilde{\Sigma}$ ) to $Z$ and $X$ (resp. $X$ and $Y$ ). Let $\phi$ and $\psi$ be the natural projections from $\widetilde{\Sigma}^{\prime} \times_{X} \widetilde{\Sigma}$ to $\widetilde{\Sigma}^{\prime}$ and $\widetilde{\Sigma}$. We call $\Sigma^{\prime \prime}$ the union of the components of $\widetilde{\Sigma}^{\prime} \times_{X} \widetilde{\Sigma}$ on which the maps $F:=f^{\prime} \circ \phi$ and $G:=g \circ \psi$ to $Z$ and $Y$ are generically of maximal rank. We consider a desingularization $\tau^{\prime \prime}: \widetilde{\Sigma}^{\prime \prime} \rightarrow \Sigma^{\prime \prime} \subset \widetilde{\Sigma^{\prime}} \times_{X} \widetilde{\Sigma}$, and call $\widetilde{F}$ and $\widetilde{G}$ the natural maps from $\widetilde{\Sigma}^{\prime \prime}$ to $Z$ and $Y$.

Lemma 1.9. - With the above notations (see also the diagram below), we have

$$
R_{\widetilde{G}}-R_{\widetilde{F}}=\left(\phi \circ \tau^{\prime \prime}\right)^{*}\left(R_{g^{\prime}}-R_{f^{\prime}}\right)+\left(\psi \circ \tau^{\prime \prime}\right)^{*}\left(R_{g}-R_{f}\right)
$$

as an equality of divisors on $\widetilde{\Sigma}^{\prime \prime}$.

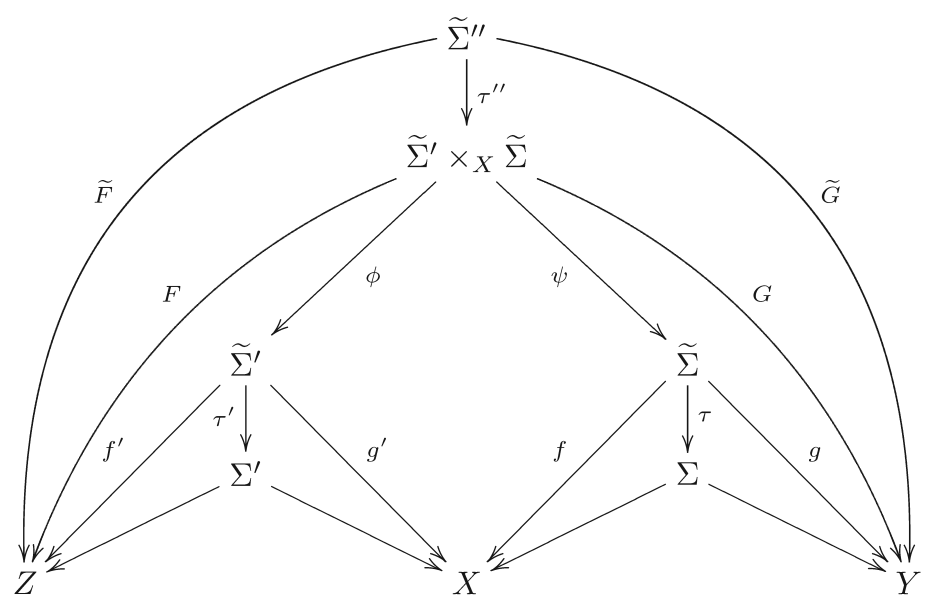


Proof. - Consider $\sigma \in \widetilde{\Sigma}^{\prime \prime}$ and let $z=\widetilde{F}(\sigma), x=g^{\prime} \circ \phi \circ \tau^{\prime \prime}(\sigma)=f \circ \psi \circ \tau^{\prime \prime}(\sigma)$, and $y=\widetilde{G}(\sigma)$. Let $\omega_{z}, \omega_{x}$ and $\omega_{y}$ be holomorphic $n$-forms, which generate respectively $K_{Z}$ near $z, K_{X}$ near $x$ and $K_{Y}$ near $y$. We then have

$$
g^{\prime *} \omega_{x}=\chi^{\prime} \cdot f^{\prime *} \omega_{z} \text { and } g^{*} \omega_{y}=\chi \cdot f^{*} \omega_{x}
$$

where $\chi^{\prime}$ is a meromorphic function defined on the inverse image $U^{\prime} \subset \widetilde{\Sigma}^{\prime}$ of a neighbourhood of $(z, x) \in Z \times X$, with divisor $\left(\chi^{\prime}\right)=\left(R_{g^{\prime}}-R_{f^{\prime}}\right) \cap U^{\prime}$, and $\chi$ is similarly a meromorphic function defined on $U \subset \widetilde{\Sigma}$ with divisor $(\chi)=\left(R_{g}-R_{f}\right) \cap U$. Pulling back these equalities on $\widetilde{\Sigma}^{\prime \prime}$ via $\phi \circ \tau^{\prime \prime}$ and $\psi \circ \tau^{\prime \prime}$, one gets

$\tau^{\prime \prime *} \phi^{*} g^{\prime *} \omega_{x}=\chi^{\prime} \circ \phi \circ \tau^{\prime \prime} \cdot \tau^{\prime \prime *} \phi^{*}{f^{\prime *}}^{\prime *} \quad$ and $\tau^{\prime \prime *} \psi^{*} g^{*} \omega_{y}=\chi \circ \psi \circ \tau^{\prime \prime} \cdot \tau^{\prime \prime *} \psi^{*} f^{*} \omega_{x}$.

Now since $g^{\prime} \circ \phi=f \circ \psi$, we have $\phi^{*} g^{\prime *} \omega_{x}=\psi^{*} f^{*} \omega_{x}$, and therefore

$$
\widetilde{G}^{*} \omega_{y}=\chi \circ \psi \circ \tau^{\prime \prime} \cdot \chi^{\prime} \circ \phi \circ \tau^{\prime \prime} \cdot \widetilde{F}^{*} \omega_{z}
$$

on the inverse image $U^{\prime \prime} \subset \widetilde{\Sigma}^{\prime \prime}$ of a neighbourhood of $(z, y) \in Z \times Y$. Note that $U^{\prime \prime}$ contains a neighbourhood of $\sigma \in \widetilde{\Sigma}^{\prime \prime}$. The meromorphic function $\chi \circ \psi \circ \tau^{\prime \prime} \cdot \chi^{\prime} \circ \phi \circ \tau^{\prime \prime}$ has divisor

$$
\left(\tau^{\prime \prime *} \phi^{*}\left(R_{g^{\prime}}-R_{f^{\prime}}\right)+\tau^{\prime \prime *} \psi^{*}\left(R_{g}-R_{f}\right)\right) \cap U^{\prime \prime} .
$$

Proof of Proposition 1.8. - Because of Proposition 1.7, to show that $\Sigma \circ \Sigma^{\prime}$ is a $\log -K$-correspondence, it only remains to prove the inequality

$$
R_{\widetilde{F}}-\widetilde{F}^{*} D_{Z} \leqslant R_{\widetilde{G}}-\widetilde{G}^{*} D_{Y}
$$

Since $\Sigma^{\prime}$ and $\Sigma$ are log- $K$-correspondences, we have

$$
R_{g^{\prime}}-R_{f^{\prime}} \geqslant g^{\prime *} D_{X}^{\prime}-f^{\prime *} D_{Z} \quad \text { and } \quad R_{g}-R_{f} \geqslant g^{*} D_{Y}-f^{*} D_{X} .
$$

By Lemma 1.9, this yields

$$
R_{\widetilde{G}}-R_{\widetilde{F}} \geqslant \tau^{\prime \prime *} \phi^{*}\left(g^{\prime *} D_{X}^{\prime}-f^{\prime *} D_{Z}\right)+\tau^{\prime \prime *} \psi^{*}\left(g^{*} D_{Y}-f^{*} D_{X}\right) .
$$

On the other hand, since $D_{X}^{\prime} \geqslant D_{X}$, and $g^{\prime} \circ \phi=f \circ \psi$, we have

$$
\phi^{*} g^{\prime *} D_{X}^{\prime}-\psi^{*} f^{*} D_{X} \geqslant 0
$$

and therefore

$$
R_{\widetilde{G}}-R_{\widetilde{F}} \geqslant \tau^{\prime \prime *} \psi^{*} g^{*} D_{Y}-\tau^{\prime \prime *} \phi^{*} f^{\prime *} D_{Z}=\widetilde{G}^{*} D_{Y}-\widetilde{F}^{*} D_{Z},
$$

which is the desired inequality. Eventually, we see from equality (9) that the morphism $J_{\widetilde{\Sigma}^{\prime \prime}}^{T}$ of line bundles on $\widetilde{\Sigma}^{\prime \prime}$ given by the log- $K$-correspondence $\Sigma \circ \Sigma^{\prime}$ 
is obtained as the composition

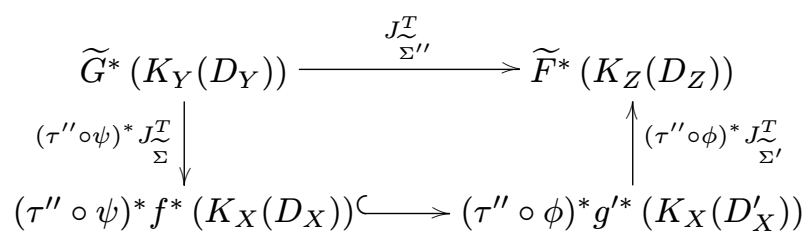

of the generalized logarithmic Jacobian maps given by $\Sigma$ and $\Sigma^{\prime}$.

\section{Intrinsic logarithmic pseudo-volume forms}

2.1. The standard Kobayashi-Eisenman pseudo-volume form. - We first recall the classical definition of the Kobayashi-Eisenman pseudo-volume form and its fundamental properties.

Let $X$ be an $n$-dimensional complex manifold. The Kobayashi-Eisenman pseudo-volume form $\Psi_{X}$ is defined by its associated Hermitian pseudo-norm on $\bigwedge^{n} T_{X}$

$$
\begin{array}{r}
\|\xi\|_{\Psi_{X, x}}=\inf \left\{\lambda>0 \text { s.t. } \exists \phi: \mathbf{D}^{n} \rightarrow X \text { with } \phi(0)=x\right. \\
\text { and } \left.\lambda \cdot J_{\phi}\left(\partial / \partial t_{1} \wedge \cdots \wedge \partial / \partial t_{n}\right)=\xi\right\}
\end{array}
$$

for $x \in X, \xi \in \bigwedge^{n} T_{X, x}$, where $\phi$ denotes a holomorphic map from the unit polydisk in $\mathbf{C}^{n}$. Note that if $\phi$ is ramified at the origin, then $J_{\phi}\left(\partial / \partial t_{1} \wedge \cdots \wedge\right.$ $\left.\partial / \partial t_{n}\right)=0$, and there does not exist any $\lambda>0$ such that $\lambda \cdot J_{\phi}\left(\partial / \partial t_{1} \wedge \cdots \wedge\right.$ $\left.\partial / \partial t_{n}\right)=\xi$ for $\xi \neq 0$.

$\Psi_{X}$ is closely related to the Poincaré hyperbolic volume form on the polydisk $\mathbf{D}^{n}$

$$
\kappa_{n}=\frac{i^{n}}{2^{n}} \bigwedge_{1 \leqslant j \leqslant n} \frac{d z_{j} \wedge d \bar{z}_{j}}{\left(1-\left|z_{j}\right|^{2}\right)^{2}} .
$$

Indeed, $\operatorname{Aut}\left(\mathbf{D}^{n}\right)$ acts transitively on the polydisk and leaves the Poincaré volume form invariant, and since the latter coincides with the standard Euclidean volume form at 0 , we find that

$$
\Psi_{X, x}=\inf \left\{\left(\phi_{b}^{-1}\right)^{*} \kappa_{n}, \phi: \mathbf{D}^{n} \rightarrow X \text { s.t. } \phi(b)=x \text { and } \phi \text { unramified } a t b\right\},
$$

where again $\phi$ runs through all holomorphic maps $\mathbf{D}^{n} \rightarrow X\left(\phi_{b}^{-1}\right.$ is the local inverse of $\phi$ near $b$ ). One also has the following result, which is a consequence of Ahlfors-Schwarz lemma (see Section 3).

Theorem 2.1 (Kobayashi). - If $X$ is isomorphic to the unit polydisk $\mathbf{D}^{n} \subset$ $\mathbf{C}^{n}$ (resp. to the quotient of $\mathbf{D}^{n}$ by a group acting freely and properly discontinuously), then the Kobayashi pseudo-volume form $\Psi_{X}$ is equal to the Poincaré 
hyperbolic volume form $\kappa_{n}$ (resp. to the hyperbolic volume form on the quotient induced by $\kappa_{n}$ ).

Finally, the following decreasing volume property is a straightforward consequence of the definition. If $Y$ is another smooth manifold of dimension $n$, and if $\phi: X \rightarrow Y$ is a holomorphic map, then we have the inequality between pseudo-volume forms on $X$

$$
\phi^{*} \Psi_{Y} \leqslant \Psi_{X}
$$

There is also a meromorphic version $\widetilde{\Psi}_{X}$ of $\Psi_{X}$, introduced by Yau in [25]. It is obtained by considering all meromorphic maps $\phi: \mathbf{D}^{n} \rightarrow X$ defined near the origin, instead of considering holomorphic maps as in the definition of $\Psi_{X}$. It is invariant under birational maps.

2.2. The Poincaré volume form on the punctured disk. - In this paragraph, we describe the Poincaré volume form on the punctured disk $\mathbf{D} \backslash\{0\}$. We will use it later on as a local model to define intrinsic pseudo-volume forms for logarithmic pairs.

The punctured disk $\mathbf{D} \backslash\{0\}$ is the quotient of $\mathbf{D}$ under the action of the subgroup of $\operatorname{Aut}(\mathbf{D})$ generated by a parabolic transformation $g \in \operatorname{Aut}(\mathbf{D})$. In order to compute the Poincaré volume form of $\mathbf{D} \backslash\{0\}$, it is however more convenient to see it as a quotient of the Poincaré upper half plane $\mathbf{H}$.

Proposition 2.2. - The Poincaré volume form of the punctured disk $\mathbf{D} \backslash\{0\}$ is

$$
\frac{i}{2} \frac{d z \wedge d \bar{z}}{|z|^{2}\left(\log |z|^{2}\right)^{2}} .
$$

Proof. - The punctured disk $\mathbf{D} \backslash\{0\}$ is the quotient of $\mathbf{H}$ by $\langle Z \mapsto Z+1\rangle$. The projection map is

$$
\pi: Z \in \mathbf{H} \mapsto z=\exp (2 \pi i Z) \in \mathbf{D} \backslash\{0\} .
$$

In particular, we have $Z=\log (z) / 2 \pi i$, and

$$
d Z=\frac{1}{2 \pi i} \frac{d z}{z} .
$$

Now, we get the Poincaré volume form on $\mathbf{D} \backslash\{0\}$ by its expression on $\mathbf{H}$

$$
\frac{i}{2} \frac{d Z \wedge d \bar{Z}}{|Z-\bar{Z}|^{2}}=\frac{i}{2} \frac{d z \wedge d \bar{z}}{|z|^{2}\left(\log |z|^{2}\right)^{2}} .
$$


Proposition 2.2 is in fact a particular case of a more general fact. Let $X$ be a punctured Riemann surface, which is universally covered by $\mathbf{D}$. Then every point $x$ in the puncture corresponds to a subgroup of $\operatorname{Aut}(\mathbf{D})$ generated by a parabolic transformation, and there is a neighbourhood of $x$ in $X$ which is isomorphic to the quotient of the circle $\{\Im(z)>1\} \subset \mathbf{H}$ by $\langle Z \mapsto Z+1\rangle$ (see e.g. [8]). Therefore, the Poincaré volume form on $X$ is given by (11) around $x$.

The Poincaré volume form on $\mathbf{D} \backslash\{0\}$ yields a logarithmic volume form on the pair $(\mathbf{D},\{0\})$, i.e. a volume form with a pole at $z=0$. It is left invariant by the log- $K$-autocorrespondences

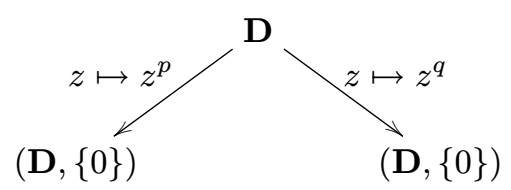

described in Example 1.5. Of course, it is also left invariant by the log- $K$ autocorrespondences of $(\mathbf{D},\{0\})$ given by the rotations $z \in \mathbf{D} \mapsto e^{i \alpha} \cdot z \in \mathbf{D}$, $\alpha \in \mathbf{R}$.

Lemma 2.3. - Up to multiplication by a constant, the Poincaré volume form on $\mathbf{D} \backslash\{0\}$ is the only logarithmic pseudo-volume form on $(\mathbf{D},\{0\})$ that is left invariant by the rotations centred at 0 and by the log-K-autocorrespondences (12).

Proof. - Indeed, let

$$
\frac{i}{2} \alpha(z) \frac{d z \wedge d \bar{z}}{|z|^{2}}
$$

be such a logarithmic volume form on the pair $(\mathbf{D},\{0\})$. Since it is invariant under the action of the rotations, $\alpha(z)$ depends only on $|z|$. On the other hand, letting $z=z^{\prime p}$, we find

$$
\frac{d z}{z}=p \frac{z^{p-1}}{z^{\prime p}} d z^{\prime}=p \frac{d z^{\prime}}{z^{\prime}} .
$$

By invariance under the action of the log- $K$-autocorrespondences (12), we find that for every $r \in] 0,1[$, and for every relatively prime integers $p$ and $q$, one has

$$
p^{2} \alpha\left(r^{p}\right)=q^{2} \alpha\left(r^{q}\right) .
$$

This implies that there exists $\lambda>0$ such that

$$
\frac{i}{2} \alpha(z) \frac{d z \wedge d \bar{z}}{|z|^{2}}=\lambda \frac{i}{2} \frac{d z \wedge d \bar{z}}{|z|^{2}\left(\log |z|^{2}\right)^{2}} .
$$


This shows in particular that the Poincaré logarithmic volume form on $(\mathbf{D},\{0\})$ is essentially characterized by its invariance under the action of log$K$-autocorrespondences of $(\mathbf{D},\{0\})$. Note that it is locally integrable around $0 \in \mathbf{D}$. We will use its $n$-dimensional version given below, to define intrinsic logarithmic pseudo-volume forms on general pairs in the next subsection.

We let $\mathbf{D}^{n}$ be the unit polydisk, with coordinates $\left(z_{1}, \ldots, z_{n}\right)$, and $\Delta_{k}$ be the divisor given by the equation $z_{n-k+1} \cdots z_{n}=0$. Then the pair $\left(\mathbf{D}^{n}, \Delta_{k}\right)$ is equipped with the Poincaré logarithmic volume form

$$
\kappa_{n, k}=\left(\frac{i}{2}\right)^{n}\left(\bigwedge_{1 \leqslant j \leqslant n-k} \frac{d z_{j} \wedge d \bar{z}_{j}}{\left(1-\left|z_{j}\right|^{2}\right)^{2}}\right) \wedge\left(\bigwedge_{n-k+1 \leqslant j \leqslant n} \frac{d z_{j} \wedge d \bar{z}_{j}}{\left|z_{j}\right|^{2}\left(\log \left|z_{j}\right|^{2}\right)^{2}}\right) .
$$

This is a $C^{\infty}$ logarithmic volume form on $\mathbf{D}^{n} \backslash \Delta_{k}$, and it is singular along $\Delta_{k}$.

2.3. Log- $K$-correspondences and intrinsic logarithmic pseudo-volume forms. - In this paragraph, we define the central object of this paper, the intrinsic pseudovolume form $\Phi_{X, D}$ of a logarithmic pair $(X, D)$.

We first need to introduce the notion of logarithmic pseudo-volume form.

Definition 2.4. - Let $(X, D)$ be a pair composed of a complex manifold $X$ of dimension $n$, and a Weil divisor $D$ of $X$. A logarithmic pseudo-volume form on $(X, D)$ is a pseudo-metric on the line bundle $\bigwedge^{n} T_{X}(-D)$.

Let $\mu$ be a logarithmic pseudo-volume form on $(X, D)$. In case it is $C^{\infty}$, it writes locally

$$
\mu=\frac{1}{|h|^{2}} \mu^{\prime},
$$

where $\mu^{\prime}$ is a $C^{\infty}$ pseudo-volume form, and $h$ is a meromorphic function with divisor $D$ : if $D=D_{1}-D_{2}$ with $D_{1}$ and $D_{2}$ non negative, then $h$ has zeroes exactly along $D_{1}$, and poles exactly along $D_{2}$. It will often be useful to allow $\mu^{\prime}$ to have singularities along the positive part $D_{1}$ of $D$ ( $c f$. definition 3.3). This is already clear from the expression of the logarithmic Poincare volume form on $(\mathbf{D},\{0\})$, given in Proposition 2.2 above, and more generally from the expression of $\kappa_{n, k}$ in (13) above.

Now $\Phi_{X, D}$ is defined as follows (we use the notations introduced in the diagram (5)).

Definition 2.5. - Let $(X, D)$ be a pair composed of an $n$-dimensional complex manifold $X$ and a normal crossing Weil divisor $D$ of $X$, such that the positive part of $D$ is reduced. For every $x \in X$, we let

$$
\begin{aligned}
\Phi_{X, D, x}= & \inf _{0 \leqslant k \leqslant n}\left(\operatorname { i n f } \left\{\left(f^{*} \kappa_{n, k}\right)_{\sigma}, \text { where } \sigma \in \widetilde{\Sigma}, \Sigma\right.\right. \text { log-K-correspondence } \\
& \text { between } \left.\left.\left(\mathbf{D}^{n}, \Delta_{k}\right) \text { and }(X, D) \text {, unramified at } \sigma \text {, with } g(\sigma)=x\right\}\right) .
\end{aligned}
$$

TOME $138-2010-\mathrm{N}^{\circ} 4$ 
A log- $K$-correspondence $\Sigma$ between $\left(\mathbf{D}^{n}, \Delta_{k}\right)$ and $(X, D)$ is said to be unramified at $\sigma$ if the inequality of divisors

$$
R_{f}-f^{*} \Delta_{k} \leqslant R_{g}-g^{*} D
$$

is an equality locally around $\sigma$. In this case, the transpose of the generalized logarithmic Jacobian yields a morphism of line bundles on $\widetilde{\Sigma}$

$$
g^{*}\left(K_{X}(D)\right) \rightarrow K_{\mathbf{D}^{n}}\left(\Delta_{k}\right)
$$

which is an isomorphism locally around $\sigma$. This authorizes the identification of $f^{*} \kappa_{n, k}$ with a Hermitian metric on $g^{*}\left(K_{X}(D)\right)^{\vee}$ locally around $\sigma$, and hence with a logarithmic pseudo-volume element at $x=g(\sigma)$.

Under the hypotheses of definition 2.5, there exists an unramified log- $K$ correspondence between $\left(\mathbf{D}^{n}, \Delta_{k}\right)$ and $(X, D)$ around every point $x \in X$ (the integer $k$ depends on $x$ ). To see this, we write $D=D_{1}-D_{2}$, with $D_{1}$ and $D_{2}$ non negative, and we choose a local holomorphic system of coordinates $\left(z_{1}, \ldots, z_{n}\right)$ centred at $x$, and defined on an open set $U \subset X$, such that $D_{2}$ is given in $U$ by $z_{1}^{l_{1}} \cdots z_{r}^{l_{r}}=0$, and $D_{1}$ is given in $U$ by $z_{n-k+1} \cdots z_{n}=0$ $(r+k \leqslant n)$. Then $D_{2}$ is the ramification divisor of the morphism $U \rightarrow V$ defined by $\left(z_{1}, \ldots, z_{n}\right) \mapsto\left(z_{1}^{l_{1}+1}, \ldots, z_{r}^{l_{r}+1}, z_{r+1}, \ldots, z_{n}\right)$, where $V$ is an open subset of $\mathbf{C}^{n}$. In particular, the graph $\Gamma$ of this morphism is an unramified log- $K$-correspondence between $\left(X, D_{1}-D_{2}\right)$ and $\left(V, D_{1}^{\prime}\right)$. Since $D_{1}^{\prime}$ is given in $V$ by the equation $z_{n-k+1} \cdots z_{n}=0$, there exists an unramified log- $K$ correspondence between $\left(\mathbf{D}^{n}, \Delta_{k}\right)$ and $\left(V, D_{1}^{\prime}\right)$. Then $\Gamma^{T} \circ \Sigma$ is an unramified $\log -K$-correspondence between $\left(\mathbf{D}^{n}, \Delta_{k}\right)$ and $(X, D)$ as we wanted. Note that $k$ is the number of branches at the point $x$ of the positive part $D_{1}$ of $D$.

Proposition 2.6. - When $D=0$, one has $\Phi_{X, 0}=\Phi_{X, a n}$. More generally, when $D$ is effective, we have

$$
\left.\Phi_{X, D}\right|_{X \backslash D}=\Phi_{X \backslash D, a n}
$$

Proof. - The reason for this is simply that the logarithmic volume form $\kappa_{n, k}$ on $\left(\mathbf{D}^{n}, \Delta_{k}\right)$ comes from the Poincaré volume form $\kappa_{n, 0}$ on $\left(\mathbf{D}^{n}, 0\right)$, due to the fact that $\mathbf{D}^{n} \backslash \Delta_{k}$ is a quotient of $\mathbf{D}^{n}$ by a group acting freely and properly discontinuously (see Paragraph 2.2). Let $\pi_{k}: \mathbf{D}^{n} \rightarrow \mathbf{D}^{n}$ be the projection corresponding to this quotient. One has by definition $\pi_{k}^{*} \kappa_{n, k}=\kappa_{n, 0}$.

Let $x$ be a point in $X \backslash D$, and $\Sigma$ be a log- $K$-correspondence between $\left(\mathbf{D}^{n}, \Delta_{k}\right)$ and $(X, D)$, with a point $\sigma \in \widetilde{\Sigma}$ above $x$, where $\Sigma$ is unramified. It yields by base change $\pi_{k}: \mathbf{D}^{n} \rightarrow \mathbf{D}^{n}$ a log- $K$-correspondence $\Sigma^{\prime}$ between 
$\left(\mathbf{D}^{n}, 0\right)$ and $(X \backslash D, 0)$. By the same base change, we get a desingularization $\widetilde{\Sigma}^{\prime}$ of $\Sigma^{\prime}$.

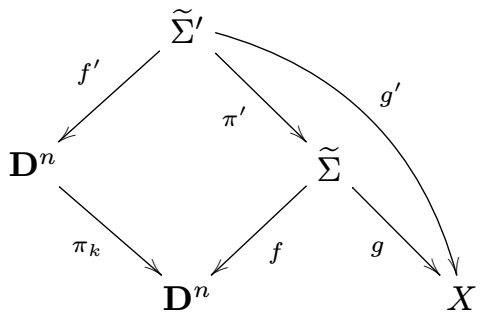

At every point $\sigma^{\prime} \in \widetilde{\Sigma}^{\prime}$ above $\sigma$, we have $\left(f^{\prime *} \kappa_{n, 0}\right)_{\sigma^{\prime}}=\left(f^{\prime *} \pi_{k}^{*} \kappa_{n, k}\right)_{\sigma^{\prime}}=$ $\pi^{\prime *}\left(f^{*} \kappa_{n, k}\right)_{\sigma}$. In particular, $\left(f^{\prime *} \kappa_{n, 0}\right)_{\sigma^{\prime}}$ and $\left(f^{*} \kappa_{n, k}\right)_{\sigma}$ yield the same logarithmic volume element at $x$. At $x$, it is therefore sufficient to take the infimum of all $f^{\prime *} \kappa_{n, 0}$ in the expression of $\Phi_{X, D}$ in definition 2.5 (i.e. we only consider $k=0)$, with $\Sigma^{\prime} \log -K$-correspondence between $\left(\mathbf{D}^{n}, 0\right)$ and $(X \backslash D, 0)$. By definition, this gives $\Phi_{X \backslash D, a n}$ at the point $x$.

When $D=-D_{2}$ is a negative divisor, a similar agument shows that $\Phi_{X,-D_{2}}$ can be computed only by looking at $\log -K$-correspondences between $\left(\mathbf{D}^{n}, 0\right)$ and $\left(X,-D_{2}\right)$. In other words, at a point $x \in X, \Phi_{X,-D_{2}}$ is the infimum of all $\left(f^{*} \kappa_{n, 0}\right)_{\sigma}$ where $\sigma \in \widetilde{\Sigma}$, and $\Sigma$ is a log-K-correspondence between $\left(\mathbf{D}^{n}, 0\right)$ and $\left(X,-D_{2}\right)$ as in definition 2.5 .

One has the following obvious comparison results.

Proposition 2.7. - Let $X$ be a complex manifold. Let $D$ and $D^{\prime}$ be normal crossing Weil divisors of $X$, such that their respective positive parts are reduced. If $D \leqslant D^{\prime}$, then one has

$$
\Phi_{X, D} \leqslant \Phi_{X, D^{\prime}}
$$

When $D=0$, one has

$$
\Phi_{X, 0}=\Phi_{X, a n} \leqslant \Psi_{X}
$$

The inequality $\Phi_{X, a n} \leqslant \Psi_{X}$ is already contained in [24]. It is a simple consequence of Example 1.2. The inequality $\Phi_{X, D} \leqslant \Phi_{X, D^{\prime}}$ when $D \leqslant D^{\prime}$ comes from the fact that a $\log -K$-correspondence between $\left(\mathbf{D}^{n}, \Delta_{k}\right)$ and $\left(X, D^{\prime}\right)$ is in particular a log- $K$-correspondence between $\left(\mathbf{D}^{n}, \Delta_{k}\right)$ and $(X, D)$.

In [24], Claire Voisin shows that if $X$ is a quotient of $\mathbf{D}^{n}$ by a group acting freely and properly discontinuously, then $\Phi_{X \text {, an }}$ is simply the Poincaré volume form on $X$. Then by Proposition 2.6, we have

$$
\Phi_{\mathbf{D}^{n}, \Delta_{k}}=\kappa_{n, k} .
$$

The proof involves curvature arguments, which are generalized in Section 3. When this is done, we will be able to show equality (14) directly ( $c f$. theorem $3.5)$. 
Lemma 2.8. - For any pair $(X, D)$ as in definition 2.5, the logarithmic pseudo-volume form $\Phi_{X, D}$ is locally integrable.

Proof. - As we already saw, the Poincaré logarithmic pseudo-volume form

$$
\frac{i}{2} \frac{d z \wedge d \bar{z}}{|z|^{2}\left(\log |z|^{2}\right)^{2}}
$$

on $(\mathbf{D},\{0\})$ is integrable at the neighbourhood of 0 . As a consequence, the Poincaré pseudo-volume form $\kappa_{n, k}$ on $\left(\mathbf{D}^{n}, \Delta_{k}\right)$ is locally integrable as well.

Now for any pair $(X, D)$ as in definition 2.5 , and for any point $x \in X$, there exists an integer $k$, and a log- $K$-isocorrespondence $\Sigma$ between $\left(\mathbf{D}^{n}, \Delta_{k}\right)$ and $(X, D)$, which is unramified at the neighbourhood of a point $\sigma \in \widetilde{\Sigma}$ over $x$. By the expression of $\Phi_{X, D}$ in definition 2.5, one then has $\left(g^{*} \Phi_{X, D}\right)_{\sigma} \leqslant\left(f^{*} \kappa_{n, k}\right)_{\sigma}$. In particular, the growth of $\Phi_{X, D}$ at $x$ is bounded from above by the growth of the Poincaré logarithmic volume form on $\left(\mathbf{D}^{n}, \Delta_{k}\right)$, and hence $\Phi_{X, D}$ is locally integrable at $x$.

An important feature of these intrinsic pseudo-volume forms is their decreasing volume properties. In the standard case, this is inequality (10). For $\Phi_{X, D}$, we even obtain an equality in the case of a proper morphism. These properties are obtained by using the study of the composition of log- $K$-correspondences, which was carried out in Paragraph 1.2. The main result is the following.

TheOREM 2.9. - Let $(X, D)$ and $\left(Y, D^{\prime}\right)$ be two pairs composed of a complex manifold and a normal crossing Weil divisor, the positive part of which is reduced. Assume there exists a log-K-correspondence $\Sigma \subset X \times Y$ between $(X, D)$ and $\left(Y, D^{\prime}\right)$. We consider a desingularization $\tau: \widetilde{\Sigma} \rightarrow \Sigma$, and use the notations of (5). Then we have the inequality of logarithmic pseudo-volume forms on $\widetilde{\Sigma}$

$$
g^{*} \Phi_{Y, D^{\prime}} \leqslant f^{*} \Phi_{X, D} .
$$

In the case when $D=D^{\prime}=0$, this is proved in [24]. As an immediate consequence of Theorem 2.9, we have the following.

Corollary 2.10. - If $\Sigma$ is a log-K-isocorrespondence between $(X, D)$ and $\left(Y, D^{\prime}\right)$, then we have the equality of logarithmic pseudo-volume forms on $\widetilde{\Sigma}$

$$
g^{*} \Phi_{Y, D^{\prime}}=f^{*} \Phi_{X, D} .
$$

Proof. - Applying Theorem 2.9 to the log- $K$-correspondence $\Sigma$ on the one hand, and to the $\log -K$-correspondence $\Sigma^{T}$ on the other hand gives both inequalities

$$
g^{*} \Phi_{Y, D^{\prime}} \leqslant f^{*} \Phi_{X, D} \text { and } f^{*} \Phi_{X, D} \leqslant g^{*} \Phi_{Y, D^{\prime}} .
$$

One then obviously has the required equality.

Applying the former corollary to Examples 1.2 and 1.3, we get the following. 
Corollary 2.11. - (i) Let $\pi: X \rightarrow Y$ be a proper morphism, with ramification divisor $R$ with normal crossings. Then we have

$$
\Phi_{X,-R}=\pi^{*} \Phi_{Y, 0} .
$$

If $\pi$ is not proper, then we only have $\Phi_{X,-R} \geqslant \pi^{*} \Phi_{Y, 0}$.

(ii) Let $\nu: Y \rightarrow X$ be a proper morphism, and $D$ be an effective, reduced, normal crossing divisor on $X$. We call $D^{\prime}$ the proper transform of $D$ with a reduced scheme structure. Then $D^{\prime}$ has normal crossings, and there exist an effective divisor $R \subset Y$, and an exceptional divisor $E \subset Y$, such that $\nu$ ramifies exactly along $\left(\nu^{*} D-D^{\prime}\right)-E+R$. If $D^{\prime}+E-R$ has normal crossings, and if $E$ is reduced, then

$$
\nu^{*} \Phi_{X, D}=\Phi_{Y, D^{\prime}+E-R}
$$

If $\nu$ is not proper, then we only have $\nu^{*} \Phi_{X, D} \leqslant \Phi_{Y, D^{\prime}+E-R}$.

Having the result of Proposition 1.8, it is fairly easy to prove the decreasing volume property. The argument is the same as in the standard case of $\Psi_{X}$.

Proof of Theorem 2.9. - Let $\sigma \in \widetilde{\Sigma}, x=f(\sigma)$, and $y=g(\sigma)$. We have to show that for every $\xi \in f^{*}\left(\bigwedge^{n} T_{X}(-D)\right)_{\sigma} \subset g^{*}\left(\bigwedge^{n} T_{Y}\left(-D^{\prime}\right)\right)_{\sigma}$, one has the inequality $\Phi_{Y, D^{\prime}}\left(g_{*} \xi\right) \leqslant \Phi_{X, D}\left(f_{*} \xi\right)$.

Let $\Sigma^{\prime} \subset \mathbf{D}^{n} \times X$ be a log- $K$-correspondence between $\left(\mathbf{D}^{n}, \Delta_{k}\right)$ and $(X, D)$, and $\sigma^{\prime} \in \widetilde{\Sigma}^{\prime}$ such that $g^{\prime}\left(\sigma^{\prime}\right)=x$, and $\Sigma^{\prime}$ is not ramified at $\sigma^{\prime}$. Then there exists $\xi^{\prime} \in g^{\prime *}\left(\bigwedge^{n} T_{X}(-D)\right)_{\sigma^{\prime}}$ satisfying $g_{*}^{\prime} \xi^{\prime}=f_{*} \xi$. By definition, $\Phi_{X, D}\left(f_{*} \xi\right)$ is the infimum of the $\kappa_{n, k}\left(f_{*}^{\prime} \xi^{\prime}\right)$, taken on all such $\Sigma^{\prime}$ and $\xi^{\prime}$.

Now $\Sigma^{\prime \prime}:=\Sigma \circ \Sigma^{\prime} \subset \mathbf{D}^{n} \times Y$ is a log- $K$-correspondence between $\left(\mathbf{D}^{n}, \Delta_{k}\right)$ and $\left(Y, D^{\prime}\right)$. It has a desingularization $\widetilde{\Sigma}^{\prime \prime}$ obtained as in the diagram (8). We use the same notations here. Since $g^{\prime}\left(\sigma^{\prime}\right)=f(\sigma)=x$, there is a point $\sigma^{\prime \prime} \in \widetilde{\Sigma}^{\prime \prime}$ above both $\sigma$ and $\sigma^{\prime}$, and there exists $\xi^{\prime \prime} \in\left(\psi \circ \tau^{\prime \prime}\right)^{*} f^{*}\left(\bigwedge^{n} T_{X}(-D)\right)_{\sigma^{\prime \prime}}=$ $\left(\phi \circ \tau^{\prime \prime}\right)^{*} g^{\prime *}\left(\bigwedge^{n} T_{X}(-D)\right)_{\sigma^{\prime \prime}}$, such that $\left(\psi \circ \tau^{\prime \prime}\right)_{*}\left(\xi^{\prime \prime}\right)=\xi$ and $\left(\phi \circ \tau^{\prime \prime}\right)_{*}\left(\xi^{\prime \prime}\right)=\xi^{\prime}$. We thus have $\widetilde{G}_{*} \xi^{\prime \prime}=g_{*} \xi$ on the one hand, and $\widetilde{F}_{*} \xi^{\prime \prime}=f_{*}^{\prime} \xi^{\prime}$, which implies that $\kappa_{n, k}\left(\widetilde{F}_{*} \xi^{\prime \prime}\right)=\kappa_{n, k}\left(f_{*}^{\prime} \xi^{\prime}\right)$ on the other hand.

Eventually, we have shown the inclusion of sets

$$
\begin{aligned}
& \left\{\kappa_{n, k}\left(f_{*}^{\prime} \xi^{\prime}\right), \text { with } \Sigma^{\prime} \subset \mathbf{D}^{n} \times X, \text { and } g_{*}^{\prime} \xi^{\prime}=f_{*} \xi\right\} \\
& \subset\left\{\kappa_{n, k}\left(f_{*}^{\prime \prime} \xi^{\prime \prime}\right), \text { with } \Sigma^{\prime \prime} \subset \mathbf{D}^{n} \times Y \text {, and } g_{*}^{\prime \prime} \xi^{\prime \prime}=g_{*} \xi\right\},
\end{aligned}
$$

where $\Sigma^{\prime}$ (resp. $\left.\Sigma^{\prime \prime}\right)$ runs through all log- $K$-correspondences between $\left(\mathbf{D}^{n}, \Delta_{k}\right)$ and $(X, D)$ (resp. $\left.\left(Y, D^{\prime}\right)\right)$. Letting $k$ take any value between 0 and $n$, and taking the infima, we get the desired inequality $\Phi_{Y, D^{\prime}}\left(g_{*} \xi\right) \leqslant \Phi_{X, D}\left(f_{*} \xi\right)$. 


\section{Curvature arguments}

Let $X$ be a complex manifold. In the case of the standard KobayashiEisenman pseudo-volume form, one has the following result, connecting the positivity (or rather the negativity) of the curvature of the canonical line bundle of $X$, and the infinitesimal measure hyperbolicity of $X$ (i.e. positivity of $\Psi_{X}$, see [6], or [23]).

THEOREM 3.1 (Kobayashi-Ochiai). - If $X$ is a projective complex manifold which is of general type, then $\Psi_{X}$ is non-degenerate outside a proper closed algebraic subset of $X$.

It is proved in [15], [10] and for $\widetilde{\Psi}_{X}$ in [25]. The main ingredient in the proof is Ahlfors-Schwarz lemma, which we generalize in this section. The converse to Theorem 3.1 is conjectured to be true in [13].

Conjecture 3.2 (Kobayashi). - If $X$ is a projective complex manifold which is not of general type, then $\Psi_{X}=0$ on a dense Zariski open subset of $X$.

We study a logarithmic variant of this conjecture in the logarithmic CalabiYau case in section 4. In this section, we first establish a generalized AhlforsSchwarz lemma in Paragraph 3.1. Then in Paragraph 3.2, we use it together with a result of Carlson and Griffiths to prove a version of the Kobayashi-Ochiai theorem relative to the logarithmic pseudo-volume forms $\Phi_{X, D}$. Note that for $D=0$, this is done in [24].

3.1. Metrics with negative curvature on $\mathbf{D}^{n}$. - We first need to recall a few facts about the logarithmic hyperbolic volume form $\kappa_{n, k}$ on $\left(\mathbf{D}^{n}, \Delta_{k}\right)$ and its curvature. It is obtained from the Poincaré metric, which has Kähler form

$$
\omega_{n, k}=\frac{i}{2}\left(\sum_{1 \leqslant j \leqslant n-k} \frac{d z_{j} \wedge d \bar{z}_{j}}{\left(1-\left|z_{j}\right|^{2}\right)^{2}}+\sum_{n-k+1 \leqslant j \leqslant n} \frac{d z_{j} \wedge d \bar{z}_{j}}{\left|z_{j}\right|^{2}\left(\log \left|z_{j}\right|^{2}\right)^{2}}\right)
$$

$\left(\kappa_{n, k}=\omega_{n, k}^{n} / n !, c f .(13)\right)$. It has Ricci form

$$
\begin{aligned}
& -i \partial \bar{\partial} \log \left(\prod_{1 \leqslant j \leqslant n-k} \frac{1}{\left(1-z_{j} \bar{z}_{j}\right)^{2}} \prod_{n-k+1 \leqslant j \leqslant n} \frac{1}{z_{j} \bar{z}_{j}\left(\log \left(z_{j} \bar{z}_{j}\right)\right)^{2}}\right) \\
& =2 i\left(\sum_{1 \leqslant j \leqslant n-k} \partial \frac{-z_{j}}{1-z_{j} \bar{z}_{j}} d \bar{z}_{j}+\sum_{n-k+1 \leqslant j \leqslant n} \partial \frac{1}{\bar{z}_{j} \log \left(z_{j} \bar{z}_{j}\right)} d \bar{z}_{j}\right)=-4 \omega_{n, k} .
\end{aligned}
$$

This gives the Kähler-Einstein equation

$$
\left(i \partial \bar{\partial} \log \kappa_{n, k}\right)^{n}=4^{n} \omega_{n, k}^{n}=4^{n} n ! \kappa_{n, k} .
$$


Eventually, we need the following definition to state properly the generalization of Ahlfors-Schwarz lemma in the logarithmic case.

Definition 3.3. - Let $(X, D)$ be a pair composed of a complex manifold $X$, and a Weil divisor $D=D^{\prime}-D^{\prime \prime}$, with $D^{\prime}$ and $D^{\prime \prime}$ non negative, and $D^{\prime}$ reduced and normal crossing. A logarithmic pseudo-volume form $\mu$ on $(X, D)$ is said to have singularities of Poincare type if it is $C^{\infty}$ on $X \backslash D^{\prime}$, and if it is equivalent to a non zero multiple of

$$
\prod_{n-k+1 \leqslant j \leqslant n} \frac{1}{\left|z_{j}\right|^{2}\left(\log \left|z_{j}\right|^{2}\right)^{2}}
$$

at the neighbourhood of $D^{\prime}$, assuming it is given by the equation $z_{n-k+1} \cdots z_{n}=$ 0 .

Of course, the logarithmic Poincaré volume form $\kappa_{n, k}$ on $\left(\mathbf{D}^{n}, \Delta_{k}\right)$ has singularities of Poincaré type.

Proposition 3.4. - Let $(X, D)$ be a pair composed of an n-dimensional complex manifold $X$ and a normal crossing Weil divisor $D$ of $X$, the positive part of which is reduced. Let $\Sigma \subset \mathbf{D}^{n} \times X$ be a log-K-correspondence between $\left(\mathbf{D}^{n}, \Delta_{k}\right)$ and $(X, D)$, with desingularization $\tau: \widetilde{\Sigma} \rightarrow \Sigma$. Let $\mu$ be a logarithmic pseudovolume form on $(X, D)$, satisfying the three following properties.

(a) $i \partial \bar{\partial} \log \mu>0$.

(b) $\mu$ has singularities of Poincaré type.

(c) $(i \partial \bar{\partial} \log \mu)^{n} \geqslant 4^{n} n ! \mu$.

Then one has the inequality of logarithmic pseudo-volume forms on $\widetilde{\Sigma}$

$$
g^{*} \mu \leqslant f^{*} \kappa_{n, k}
$$

Note that if $D$ has a positive part, then $\mu$ has poles, and therefore inequality (c) cannot be true if $\mu$ has no singularity, i.e. if it writes locally $\mu^{\prime} /|h|^{2}$, where $h$ is a meromorphic function with divisor $D$, and $\mu^{\prime}$ is a $C^{\infty}$ pseudo-volume form on $X$. In this case, $i \partial \bar{\partial} \log \mu=i \partial \bar{\partial} \log \mu^{\prime}$ is a $C^{\infty}(1,1)$-form. In particular, it has no pole on $X$.

Proof of Proposition 3.4. - We begin by restricting $\Sigma$ to $\mathbf{D}_{1-\varepsilon}^{n}:=\mathbf{D}(0,1-\varepsilon)^{n}$ as follows. We let $\Sigma_{\varepsilon}$ be the inverse image of $\Sigma$ via the map $((1-\varepsilon)$ id,id $)$ : $\mathbf{D}^{n} \times X \rightarrow \mathbf{D}^{n} \times X$. This would correspond in the morphism case to the transformation of $\varphi: \mathbf{D}^{n} \rightarrow X$ into $\widetilde{\varphi}:=\varphi_{\mid \mathbf{D}_{1-\varepsilon}^{n}}((1-\varepsilon) \times \cdot): \mathbf{D}^{n} \rightarrow X$. Of course one gets from $\widetilde{\Sigma}$ a desingularization $\widetilde{\Sigma}_{\varepsilon}$ of $\Sigma_{\varepsilon}$ in a natural way, with maps $f_{\varepsilon}$ and $g_{\varepsilon}$ to $\mathbf{D}^{n}$ and $X$. 
Next, one considers the ratio

$$
\psi_{\varepsilon}:=\frac{g_{\varepsilon}^{*} \mu}{f_{\varepsilon}^{*} \kappa_{n, k}} .
$$

It is a non-negative $C^{\infty}$-function on $\widetilde{\Sigma}_{\varepsilon}$. To see why, we first write locally $\mu=\mu^{\prime} /|h|^{2}$, where $h$ is a meromorphic function with divisor $D$ (i.e. it has zeroes along the positive part $D_{1}$ of $D$, and poles along the negative part $D_{2}$ of $D)$, and $\mu^{\prime}=\chi_{\mu^{\prime}}(i / 2)^{n} \bigwedge_{1 \leqslant j \leqslant n} d z_{j} \wedge d \bar{z}_{j}$ is a pseudo-volume form on $X$, which is $C^{\infty}$ on $X \backslash D_{1}$, and singular along $D_{1}$, so that $\mu$ has singularities of Poincaré type. On the other hand, we let similarly

$$
\chi_{n, k}=\left(\prod_{1 \leqslant j \leqslant n-k}\left(1-\left|t_{j}\right|^{2}\right)^{-2}\right)\left(\prod_{n-k+1 \leqslant j \leqslant n}\left(\log \left|t_{j}\right|^{2}\right)^{-2}\right),
$$

so that $\kappa_{n, k}=\chi_{n, k}(i / 2)^{n} \bigwedge_{1 \leqslant j \leqslant n} d t_{j} \wedge d \bar{t}_{j} /\left|t_{n-k+1} \cdots t_{n}\right|^{2}$. We let $h_{k}(t)=$ $t_{n-k+1} \cdots t_{n}$ be a holomorphic equation of $\Delta_{k} \subset \mathbf{D}^{n}$. Then $\psi_{\varepsilon}$ writes locally

$$
\psi_{\varepsilon}=\frac{\left|s_{g}\right|^{2} g_{\varepsilon}^{*} \chi_{\mu^{\prime}}}{\left|g_{\varepsilon}^{*} h\right|^{2}} \cdot \frac{\left|f_{\varepsilon}^{*} h_{k}\right|^{2}}{\left|s_{f}\right|^{2} f_{\varepsilon}^{*} \chi_{n, k}},
$$

where $s_{f}$ and $s_{g}$ are local analytic equations of the ramification divisors $R_{f}$ and $R_{g}$ respectively. Now since $\Sigma$ is a log-K-correspondence between $\left(\mathbf{D}^{n}, \Delta_{k}\right)$ and $(X, D)$, one has $R_{g}-g^{*} D \geqslant R_{f}-f^{*} \Delta_{k}$, and therefore $s_{g} \cdot f_{\varepsilon}^{*} h_{k} / s_{f} \cdot g_{\varepsilon}^{*} h$ is $C^{\infty}$ on $X$. In addition, since $\mu$ has singularities of Poincaré type, the ratio $g_{\varepsilon}^{*} \chi_{\mu^{\prime}} / f_{\varepsilon}^{*} \chi_{n, k}$ is $C^{\infty}$ on $X$ as well.

Now, $1 / \chi_{n, k}$ tends to 0 on the boundary of $\mathbf{D}^{n}$, while $\psi_{\varepsilon} \cdot f_{\varepsilon}^{*} \chi_{n, k}$ stays bounded near the boundary of $\widetilde{\Sigma}_{\varepsilon}$, since $\overline{\mathbf{D}}_{1-\varepsilon}^{n}$ is compact and $f$ is proper. This gives

$$
\lim _{f_{\varepsilon}(x) \rightarrow \partial \mathbf{D}^{n}} \psi_{\varepsilon}(x)=0,
$$

and so by properness of $f_{\varepsilon}$, the ratio $\psi_{\varepsilon}$ has a maximum on $\widetilde{\Sigma}_{\varepsilon}$. Let $x_{0} \in \widetilde{\Sigma}_{\varepsilon}$ be a point where this maximum is reached, and write $c:=\psi_{\varepsilon}\left(x_{0}\right)$. Then we just have to show that $c \leqslant 1$ (we will then get the proposition by letting $\varepsilon$ tend to $0)$.

We now suppose that $c>1$, and show as in the standard proof that this contradicts hypotheses (a), (b) and (c). For $\alpha \in] 1, c[$, define

$$
\widetilde{\Sigma}_{\varepsilon, \alpha}=\left\{x \in \widetilde{\Sigma}_{\varepsilon} \text { s.t. } \psi_{\varepsilon}(x) \geqslant \alpha\right\} .
$$

$\psi_{\varepsilon}(x)$ tends to 0 near the boundary of $\widetilde{\Sigma}_{\varepsilon}$, so $\widetilde{\Sigma}_{\varepsilon, \alpha}$ is compact, and with smooth boundary for $\alpha$ generic. Since $i \partial \bar{\partial} \log \chi_{\mu^{\prime}}=i \partial \bar{\partial} \log \mu>0$, and $i \partial \bar{\partial} \log \chi_{n, k}=$ $4 \omega_{n, k}>0$,

$$
\begin{array}{r}
\theta:=g_{\varepsilon}^{*}\left(i \partial \bar{\partial} \log \chi_{\mu^{\prime}}\right)^{n-1}+g_{\varepsilon}^{*}\left(i \partial \bar{\partial} \log \chi_{\mu^{\prime}}\right)^{n-2} f_{\varepsilon}^{*}\left(i \partial \bar{\partial} \log \chi_{n, k}\right) \\
+\cdots+f_{\varepsilon}^{*}\left(i \partial \bar{\partial} \log \chi_{n, k}\right)^{n-1}
\end{array}
$$


is a semi-positive $(n-1, n-1)$-form, positive away from the positive part of $R_{f}-f^{*} \Delta_{k}$. Note that it is $+\infty$ along the negative part of $R_{g}-g^{*} D$, i.e. along the positive part of the reduced divisor $\left(g^{*} D\right)_{\text {red }}$, where $\mu^{\prime}$ is singular. Now $\psi_{\varepsilon}$ has Laplacian $i \partial \bar{\partial} \log \psi_{\varepsilon}=i \partial \bar{\partial} \log \left(g_{\varepsilon}^{*} \chi_{\mu^{\prime}}\right)-i \partial \bar{\partial} \log \left(f_{\varepsilon}^{*} \chi_{n, k}\right)$, and we have

$$
\begin{aligned}
\left(i \partial \bar{\partial} \log \psi_{\varepsilon}\right) \theta & =g_{\varepsilon}^{*}\left(i \partial \bar{\partial} \log \chi_{\mu^{\prime}}\right)^{n}-f_{\varepsilon}^{*}\left(i \partial \bar{\partial} \log \chi_{n, k}\right)^{n} \\
& \geqslant 4^{n} n !\left(g_{\varepsilon}^{*} \mu-f_{\varepsilon}^{*} \kappa_{n, k}\right),
\end{aligned}
$$

where the inequality is given by condition (c) on $\mu$, and by the Kähler-Einstein equation (15) for the hyperbolic volume form. Now in $\widetilde{\Sigma}_{\varepsilon, \alpha}$ we have $\psi_{\varepsilon}>1$, and therefore

$$
g_{\varepsilon}^{*} \mu-f_{\varepsilon}^{*} \kappa_{n} \geqslant 0
$$

with strict inequality away from the positive part of $R_{f}-f^{*} \Delta_{k}$ (again, this is $+\infty$ along the positive part of $\left.\left(g^{*} D\right)_{\text {red }}\right)$. Finally the Laplacian $i \partial \bar{\partial} \log \psi_{\varepsilon}$ is semi-positive, and positive away from the positive part of $R_{f}-f^{*} \Delta_{k}$.

When $x_{0}$ does not belong to the positive part of $R_{f}-f^{*} \Delta_{k}$, one can conclude from the maximum principle for pluri-subharmonic functions that $\psi_{\varepsilon}$ cannot have a maximum at $x_{0}$, which is a contradiction, and proves $c \leqslant 1$ as we wanted. Otherwise, one has to apply the following standard argument. One chooses $m$ satisfying $\log \alpha<m<\log c$, and then defines a function

$$
\mu^{+}(x)=\max \left(0, \log \psi_{\varepsilon}(x)-m\right) .
$$

It is non-negative, positive at $x_{0}$, and vanishes identically near the boundary $\partial \widetilde{\Sigma}_{\varepsilon, \alpha}$. Therefore we have

$$
\int_{\widetilde{\Sigma}_{\varepsilon, \alpha}} \mu^{+}\left(i \partial \bar{\partial} \log \psi_{\varepsilon}\right) \theta>0
$$

Note that the form $\left(i \partial \bar{\partial} \log \psi_{\varepsilon}\right) \theta$ is indeed integrable in $\widetilde{\Sigma}_{\varepsilon, \alpha}$, because both $\mu$ and $\kappa_{n, k}$ only have singularities of Poincaré type. The derivatives of $\mu^{+}$are integrable, so we can integrate by parts the previous integral. This gives

$$
-\int_{\widetilde{\Sigma}_{\varepsilon, \alpha}} \partial \mu^{+} \wedge\left(i \bar{\partial} \log \psi_{\varepsilon}\right) \theta>0 .
$$

Since $\mu^{+}=\log \psi_{\varepsilon}-m$ when it is non-zero, the former inequality gives

$$
\int_{\widetilde{\Sigma}_{\varepsilon, \alpha}} \partial \mu^{+} \wedge\left(i \bar{\partial} \log \psi_{\varepsilon}\right) \theta=\int_{\left\{\log \psi_{\varepsilon}(x) \geqslant m\right\}} i\left(\partial \log \psi_{\varepsilon}\right) \wedge\left(\bar{\partial} \log \psi_{\varepsilon}\right) \theta<0,
$$

which is a contradiction, since the right-hand side integral is obviously positive.

As a first application of this result, we can show directly that $\Phi_{\mathbf{D}^{n}, \Delta_{k}}$ is indeed given by the hyperbolic logarithmic Poincaré volume form (see Proposition 2.6 as well).

TOME $138-2010-\mathrm{N}^{\mathrm{O}} 4$ 
TheOREm 3.5. - For $0 \leqslant k \leqslant n$, we have the equality of logarithmic volume forms on $\left(\mathbf{D}^{n}, \Delta_{k}\right)$

$$
\Phi_{\mathbf{D}^{n}, \Delta_{k}}=\kappa_{n, k} .
$$

Proof. - The diagonal in $\mathbf{D}^{n} \times \mathbf{D}^{n}$ is a log- $K$-correspondence between $\left(\mathbf{D}^{n}, \Delta_{k}\right)$ and itself. By definition 2.5, we thus have $\Phi_{\mathbf{D}^{n}, \Delta_{k}} \leqslant \kappa_{n, k}$.

On the other hand, let $\Sigma$ be a $\log -K$-correspondence between $\left(\mathbf{D}^{n}, \Delta_{p}\right)$ and $\left(\mathbf{D}^{n}, \Delta_{k}\right)$, with $0 \leqslant p \leqslant n$. Then by Proposition 3.4, and using the standard notations of (5), we have $g^{*} \kappa_{n, k} \leqslant f^{*} \kappa_{n, p}$. This implies that $\kappa_{n, k} \leqslant \Phi_{\mathbf{D}^{n}, \Delta_{k}}$.

3.2. Mappings onto pairs with positive logarithmic canonical bundle. - Before we state our generalization of the Kobayashi-Ochiai theorem 3.1, let us first recall that a variety $X$ is said to be of general type if the canonical bundle $K_{X}$ is big. A line bundle $L$ on $X$ is said to be big if it has maximal Iitaka-Kodaira dimension $\kappa(X, L)=\operatorname{dim} X$. This is equivalent to the fact, that the image of the rational map associated to the linear system $|m L|$ is of maximal dimension $\operatorname{dim} X$, for $m$ big enough and divisible enough (see e.g. [20]). Given a Weil divisor $D$ on $X$, the condition corresponding to the fact that $X$ is of general type is in our case the bigness of $K_{X}+D$. However, we shall need some slightly stronger hypotheses to prove our result.

TheOREM 3.6. - Let $(X, D)$ be a pair composed of a projective $n$-dimensional complex manifold $X$, and a normal crossing Weil divisor $D$ of $X$, the positive part of which is reduced and has global normal crossings. If $K_{X}+D$ is ample, then $\Phi_{X, D}>0$ away from a proper closed algebraic subset of $X$.

As an important consequence of this result, we can bound from above the degree of a morphism of logarithmic pairs, which is onto a pair with positive logarithmic canonical bundle.

Corollary 3.7. - Let $(X, D)$ be a pair composed of a projective manifold $X$, and a normal crossing Weil divisor $D$, the positive part of which is reduced, and has global normal crossings. We assume that $K_{X}+D$ is ample. Let $\left(Y, D^{\prime}\right)$ be another logarithmic pair. We assume that $D^{\prime}$ has normal crossings, and that its positive part is reduced. For every dominant morphism $\phi:\left(Y, D^{\prime}\right) \rightarrow(X, D)$, we have

$$
\operatorname{deg} \phi \leqslant \frac{\int_{Y} \Phi_{Y, D^{\prime}}}{\int_{X} \Phi_{X, D}}
$$


Proof. - The morphism $\phi$ induces a log- $K$-correspondence between $\left(Y, D^{\prime}\right)$ and $(X, D)$ (cf. Example 1.3). By decreasing volume property, we thus have

$$
\phi^{*} \Phi_{X, D} \leqslant \Phi_{Y, D^{\prime}} .
$$

Since for $0 \leqslant k \leqslant n$, the volume form $\kappa_{n, k}$ is integrable at the neighbourhood of $\Delta_{k}$, and since $X$ and $Y$ are compact, definition 2.5 implies that the pseudovolume forms $\Phi_{X, D}$ and $\Phi_{Y, D^{\prime}}$ are integrable on $X$ and $Y$ respectively. In particular, (16) yields

$$
(\operatorname{deg} \phi) \int_{X} \Phi_{X, D} \leqslant \int_{Y} \Phi_{Y, D^{\prime}}
$$

Now, by Theorem 3.6, the integral $\int_{X} \Phi_{X, D}$ is positive, so $\operatorname{deg} \phi$ is bounded from above.

Along with Ahlfors-Schwarz Lemma 3.4, the main ingredient in the proof of Theorem 3.6 above is the following result.

Lemma 3.8 (Carlson-Griffiths, [4]). — Let $(X, D)$ be a pair as in Theorem 3.6. Then there exists a logarithmic volume form with Poincaré singularities $\mu$ on $(X, D)$, such that $i \partial \bar{\partial} \log \mu>0$ and $(i \partial \bar{\partial} \log \mu)^{n} \geqslant \mu$.

For a complete proof of this, we refer to [11], Proposition 2.17. Let us still explain how this pseudo-volume form is constructed. We choose a Hermitian metric $h_{0}$ on $K_{X}$. Equivalently, $h_{0}^{-1}$ is a $C^{\infty}$ volume form $\mu_{X}$ on $X$. We assume for simplicity that $D$ is effective, the general case follows easily. Then $D$ is assumed to have global normal crossings, so it writes $D=D_{1}+\cdots+D_{p}$, where the $D_{j}$ are smooth divisors on $X$, meeting transversaly. We choose a Hermitian metric $h_{j}$ for every line bundle $\theta_{X}\left(D_{j}\right)$, and we let $s_{j}$ be a global section of this line bundle, with zero divisor $D_{j}$. Then for $\alpha>0$ sufficiently small, the pseudo-volume form

$$
\mu_{\alpha}:=\frac{\mu_{X}}{\prod_{j=1}^{p} h_{j}\left(s_{j}\right)\left(\log \left(\alpha h_{j}\left(s_{j}\right)\right)^{2}\right.}
$$

satisfies the required properties.

Before we continue, we want to underline that this result is used as a first step in the construction of complete Kähler-Einstein metrics with negative Ricci curvature on the complement of hypersurfaces of projective algebraic manifolds (see [12] and [19]).

Proof of Theorem 3.6. - By Lemma 3.8, there exists a logarithmic pseudovolume form $\mu$ on $(X, D)$, with Poincaré singularities, and such that $i \partial \bar{\partial} \log \mu>$ 0 , and $(i \partial \bar{\partial} \log \mu)^{n} \geqslant \mu$. Up to a rescaling, one can assume that $\mu$ satisfies 
hypothesis (c) of Proposition 3.4. Then if $0 \leqslant k \leqslant n$, and for every log$K$-correspondence $\Sigma$ between $\left(\mathbf{D}^{n}, \Delta_{k}\right)$ and $(X, D)$, we have the inequality of pseudo-volume forms on a desingularization $\widetilde{\Sigma}$

$$
g^{*} \mu \leqslant f^{*} \kappa_{n, k} .
$$

It thus follows from the definition 2.5 of $\Phi_{X, D}$ that $\Phi_{X, D} \geqslant g^{*} \mu$, which implies that $\Phi_{X, D}$ is positive on a Zariski dense open subset of $X$, since $\mu$ is a true logarithmic volume form on $(X, D)$.

\section{Log- $K$-autocorrespondences on log- $K$-trivial pairs}

In this section, we prove that for many pairs $(X, D)$, where $X$ is a complex manifold, and $D$ an effective divisor on $X$, which is reduced and has normal crossings, such that the line bundle $K_{X}(D)$ is trivial, the pseudo-volume form $\Phi_{X, D}$ vanishes. This can be interpreted as a special case of the Kobayashi Conjecture 3.2 in the logarithmic case. Again, the case $D=0$ is handled in [24]. However, one has to be slightly more careful in our case for the proof. This is due to the fact that we need to pull-back differential forms via correspondences, instead of cohomology classes.

4.1. Log- $K$-autocorrespondences and the Kobayashi conjecture. - In view of Theorem 3.6, which has to be seen as a logarithmic version of the KobayashiOchiai theorem for $\Phi_{X, D}$, the Kobayashi conjecture generalizes as follows in the logarithmic case.

ConjeCture 4.1. - Let $(X, D)$ be a pair composed of a projective manifold $X$ and a normal crossing Weil divisor $D \subset X$, the positive part of which is reduced. If $(X, D)$ is not of log-general type (i.e. if $K_{X}+D$ is not big), then $\Phi_{X, D}$ vanishes on a dense Zariski open subset of $X$.

This section is devoted to the proof of the following result, which goes in the direction of this conjecture.

THEOREM 4.2. - Let $(X, D)$ be a pair consisting of a smooth projective variety $X$, and an effective divisor $D \subset X$, which is reduced and has normal crossings, such that $K_{X}(D)$ is trivial. Assume that there exists a smooth, rationally connected variety $Y$, such that $X$ can be realized as a hypersurface $X \subset Y, D=X \cap X^{\prime}$, where $X^{\prime} \subset Y$ is a reduced hypersurface such that $X+X^{\prime} \in\left|-K_{Y}\right|$. Then $\Phi_{X, D}=0$. 
Note that this shows the log-Kobayashi conjecture for a very wide class of log- $K$-trivial pairs.

The next proposition shows why this theorem is an easy consequence of the existence on such pairs of log- $K$-autocorrespondences (i.e. log- $K$ isocorrespondences between a pair $(X, D)$ and itself, see definition 1.4) satisfying a certain dilation property.

Proposition 4.3. - Let $(X, D)$ be a pair composed of a smooth projective variety $X$, and an effective divisor $D \subset X$, which is reduced and has normal crossings, such that $K_{X}(D)$ is trivial. Let $\eta_{X}$ be a generator of $\mathrm{H}^{0}\left(X, K_{X}(D)\right)$. If there exists a log-K-autocorrespondence $\Sigma$ of the pair $(X, D)$, such that for a desingularization $\tau: \widetilde{\Sigma} \rightarrow \Sigma$, and with the notations (5) of definition 1.1, one has

$$
f^{*} \eta_{X}=\lambda g^{*} \eta_{X}
$$

where $\lambda$ is a complex number with $|\lambda| \neq 1$, then $\Phi_{X, D}=0$.

Proof. - Let $\Omega_{X, D}$ be defined as

$$
\Omega_{X, D}=(-1)^{\frac{n(n-1)}{2}} i^{n} \eta_{X} \wedge \bar{\eta}_{X}
$$

The dilation property satisfied by $\Sigma$ shows that

$$
f^{*} \Omega_{X, D}=|\lambda|^{2} g^{*} \Omega_{X, D},
$$

while Corollary 2.10 gives the equality of pseudo-volume forms

$$
f^{*} \Phi_{X, D}=g^{*} \Phi_{X, D} .
$$

Now there exists a bounded, upper semi-continuous function $\chi$ on $X$, such that $\Phi_{X, D}=\chi \Omega_{X, D} \cdot \chi$ has a maximum on $X$. Let $x$ be a point on $X$ where $\chi(x)$ is this maximum. Then take $\sigma \in \widetilde{\Sigma}$ such that $f(\sigma)=x$, and let $y=g(\sigma)$. Equality (17) eventually gives $\chi(y)=|\lambda|^{2} \chi(x)$. Since of course we can assume $|\lambda|>1$ by symmetry, this shows that $\chi(x)=0$, and hence that $\chi=0$ as we wanted.

Now the proof of 4.2 amounts to show the following theorem. This is done in the remainder of this section.

THEOREM 4.4. - If $(X, D)$ is a pair satisfying the condition of theorem 4.2, then there exists a log-K-autocorrespondence of $(X, D)$, such that (with the notations of proposition 4.3)

$$
m f^{*} \eta_{X}=-m^{\prime} g^{*} \eta_{X}
$$

where $m$ and $m^{\prime}$ are distinct positive integers.

In fact, by the following remark, it is enough to prove that there exists a proper 0-correspondence $\Sigma \subset X \times X$ (cf. definition 1.6) satisfying relation (18).

TOME $138-2010-\mathrm{N}^{\mathrm{O}} 4$ 
REMARK 4.5. - Let $\Sigma \subset X \times X$ be a reduced closed analytic subset, with both projections proper and generically finite. If $m f^{*} \eta_{X}=-m^{\prime} g^{*} \eta_{X}$ (with the notations of proposition 4.3), then $\Sigma$ is a $\log -K$-autocorrespondence of $(X, D)$.

Indeed, $\eta_{X}$ is an everywhere non zero section of $K_{X}(D)$, so the meromorphic $n$-form $f^{*} \eta_{X}\left(\right.$ resp. $\left.g^{*} \eta_{X}\right)$ on $\widetilde{\Sigma}$ has divisor $R_{f}-f^{*} D\left(\right.$ resp. $\left.R_{g}-g^{*} D\right)$, i.e. it has zeroes along $R_{f}$, and poles along $f^{*} D$. Equality (18) thus yields the equality of divisors

$$
R_{f}-f^{*} D=R_{g}-g^{*} D
$$

on the desingularization $\widetilde{\Sigma}$, proving that $\Sigma$ is a $\log$ - $K$-autocorrespondence of $(X, D)$.

4.2. Geometric construction of self-correspondences on $K$-trivial pairs. - From now on, we let $Y$ be a smooth, rationally connected variety of dimension $n+1$, and $\bar{X} \subset Y$ be a reduced hypersurface in the anticanonical linear class of $Y$ (i.e. $\left.\bar{X} \in\left|-K_{Y}\right|\right)$, with smooth locus $\operatorname{sm}(\bar{X}) \subset \bar{X}$. By adjunction, $\bar{X}$ has a canonical sheaf $K_{\bar{X}}$, and it is trivial. We construct in this section a 0 -correspondence $\Sigma \subset \bar{X} \times \bar{X}$. In [24], such correspondences were constructed in the case of smooth $\bar{X}$, and proved to be $K$-autocorrespondences. The process is in some way analogous to the geometric definition of addition on an elliptic curve in $\mathbf{P}^{2}$. Of course, we have in mind the case when $\bar{X}=X \cup X^{\prime}$ is the reunion of two reduced hypersurfaces, $X$ smooth, and $D:=X \cap X^{\prime}$ is reduced and has normal crossings, which will give theorem 4.4 for the pair $(X, D)$, where $D$ is seen as a divisor of $X$.

Since $Y$ is rationally connected, there exists a rational curve $C_{0} \subset Y$ satisfying the two following conditions.

(i) $C_{0}$ does not meet the singular part of $\bar{X}$, and $C_{0} \cap \bar{X}=m x_{0}+m^{\prime} y_{0}+z_{0}$ as a divisor on $C_{0}$, where $x_{0}$ and $y_{0}$ are distinct points of $\operatorname{sm}(\bar{X}), m$ and $m^{\prime}$ are (fixed) distinct positive integers, and $z_{0}$ is a reduced 0 -cycle on $C_{0}$, disjoint from $x_{0}$ and $y_{0}$.

(ii) The deformations of the subscheme $C_{0} \subset Y$ induce arbitrary deformations of the $M$-jet of $C_{0}$ at two points of intersection with $\bar{X}$, where $M:=$ $\max \left(m, m^{\prime}\right)$.

This is given by the fact that $C_{0}$ can be chosen with arbitrarily ample normal bundle, because $Y$ is rationally connected (see [16]).

In addition, one chooses a hypersurface $W \subset \bar{X}$ containing the 0-cycle $z_{0}$. We denote by $\left|C_{0}\right|$ the space parametrizing all deformations of the subscheme $C_{0} \subset Y$. Now define $\Sigma_{\text {tot }} \subset \operatorname{sm}(\bar{X}) \times \operatorname{sm}(\bar{X}) \times\left|C_{0}\right|$ by

$$
\begin{aligned}
\Sigma_{\text {tot }}:= & \left\{(x, y, C) \text { s.t. } C \text { deformation of } C_{0},\right. \\
& \left.C \cap \bar{X}=m x+m^{\prime} y+z, \operatorname{supp}(z) \subset W\right\} .
\end{aligned}
$$


Proposition 4.6. - For a generic choice of $W$, the Zariski closure of $\Sigma_{\text {tot }}$ in $\bar{X} \times \bar{X} \times\left|C_{0}\right|$ has a unique $n$-dimensional irreducible component through the point $\left(x_{0}, y_{0}, C_{0}\right)$. We call this component $\Sigma^{\prime}$. The Zariski closure of the projection of $\Sigma^{\prime}$ in $\bar{X} \times \bar{X}$ is irreducible of dimension $n$. Let us denote it $\Sigma$. Then $\Sigma \subset \bar{X} \times \bar{X}$ is a 0 -correspondence.

We call $f^{\prime}$ and $g^{\prime}$ the two morphisms $\Sigma^{\prime} \rightarrow \bar{X}$ given by the projections of $\bar{X} \times \bar{X} \times\left|C_{0}\right|$ on its first and second factors respectively. $\Sigma$ is an $n$-dimensional component of the closure of $\left(f^{\prime}, g^{\prime}\right)\left(\Sigma^{\prime}\right)$.

Proof. - The proposition essentially follows from the fact that, for a generic choice of $W, \Sigma_{\text {tot }}$ is smooth and of dimension $n=\operatorname{dim} \bar{X}$ at the point $\left(x_{0}, y_{0}, C_{0}\right)$. This gives the existence of a unique component $\Sigma^{\prime}$ at once. On the other hand, since the deformations of $C_{0} \subset Y$ induce arbitrary deformations of the $M$-jet of $C_{0}$ at $x_{0}$ and $y_{0}$, this implies that the image $\left(f^{\prime}, g^{\prime}\right)\left(\Sigma^{\prime}\right) \subset \bar{X} \times \bar{X}$ has a component of dimension $n$.

So let us show that $\Sigma_{\text {tot }}$ is smooth and of dimension $n$ near $\left(x_{0}, y_{0}, C_{0}\right)$. We first study the Hilbert scheme of $C_{0} \subset Y$ at the infinitesimal neighbourhood of $C_{0}$. Since $C_{0}$ is a rational curve, and its normal bundle $N_{C_{0} / Y}$ is ample, we have $\mathrm{h}^{1}\left(C_{0}, N_{C_{0} / Y}\right)=0$, and the Hilbert scheme of $C_{0} \subset Y$ is smooth, and of dimension

$$
h^{0}\left(C_{0}, N_{C_{0} / Y}\right)=\chi\left(C_{0}, N_{C_{0} / Y}\right) .
$$

By the Riemann-Roch formula,

$$
\begin{aligned}
\chi\left(C_{0}, N_{C_{0} / Y}\right) & =\operatorname{deg}\left(N_{C_{0} / Y}\right)+\operatorname{rg}\left(N_{C_{0} / Y}\right)(1-g) \\
& =-K_{Y} \cdot C_{0}+(2 g-2)+n(1-g)=n-2-K_{Y} \cdot C_{0}
\end{aligned}
$$

$\left(g=0\right.$ is the geometric genus of $\left.C_{0}\right)$. Now to compute the dimension of $\Sigma_{\text {tot }}$, we note that we impose to the deformations $C$ of $C_{0}$ to meet $\bar{X}$ properly and in the smooth locus $\operatorname{sm}(\bar{X})$ (this is open), and to have intersection $C \cap \bar{X}=$ $m x+m^{\prime} y+z$, with $\operatorname{supp}(z) \subset W$. This imposes at most $(m-1)+\left(m^{\prime}-\right.$ $1)+\operatorname{deg} z$ conditions. In fact, for a generic choice of $W$, these conditions are infinitesimally independent at the starting point $\left(x_{0}, y_{0}, C_{0}\right)$. We deduce from this that $\Sigma_{\text {tot }}$ is smooth at the neighbourhood of $\left(x_{0}, y_{0}, C_{0}\right)$, and, since $\bar{X} \in\left|-K_{Y}\right|$, of dimension

$$
\left(-K_{Y} \cdot C_{0}+n-2\right)-\left(m+m^{\prime}-2+\operatorname{deg} z\right)=n-K_{Y} \cdot C_{0}-\bar{X} \cdot C_{0}=n .
$$

4.3. Realization as log- $K$-autocorrespondences. - When $\bar{X}=X \cup X^{\prime}$ is the union of two reduced hypersurfaces, and if $X$ is smooth, then a section of $K_{\bar{X}}$ gives a meromorphic $n$-form on the component $X$ by restriction to the smooth locus of $\bar{X}$. In this subsection, we show the following. 
TheOREM 4.7. - Let $\Sigma \subset \bar{X} \times \bar{X}$ be as before (see proposition 4.6). We consider a desingularization $\tau: \widetilde{\Sigma} \rightarrow \Sigma$ and use the notations (5) of definition 1.1. Let $\eta_{\bar{X}}$ be a generator of $\mathrm{H}^{0}\left(\bar{X}, K_{\bar{X}}\right)$ (recall that $K_{\bar{X}}$ is trivial). We have the equality of meromorphic differential $n$-forms on $\widetilde{\Sigma}$

$$
m f^{*} \eta_{\bar{X}}+m^{\prime} g^{*} \eta_{\bar{X}}=0
$$

Specializing this result to the case $\bar{X}=X \cup X^{\prime}$ already mentioned, we get the following corollary. Of course, it proves theorem 4.4, and because of Proposition 4.3, we get our main Theorem 4.2 ( $c f$. subsection 4.1).

Corollary 4.8. - If $\bar{X}=X \cup X^{\prime}$ is the union of two reduced hypersurfaces, $X$ smooth, and $D:=X \cap X^{\prime}$ reduced, then $\Sigma$ induces a 0 -correspondence $\Sigma_{1}:=$ $\Sigma \cap(X \times X) \subset X \times X$. If $\eta_{X}$ is a generator of $\mathrm{H}^{0}\left(X, K_{X}(D)\right)$, then we have the equality of meromorphic differential $n$-forms

$$
m f_{1}^{*} \eta_{X}+m^{\prime} g_{1}^{*} \eta_{X}=0
$$

on a desingularization $\widetilde{\Sigma}_{1} \rightarrow \Sigma_{1}$, where $f_{1}$ and $g_{1}$ are the natural morphisms $\Sigma_{1} \rightarrow X$. In particular, $\Sigma_{1}$ is a log-K-autocorrespondence of the pair $(X, D)$.

Proof. - In this case, $\Sigma \subset \bar{X} \times \bar{X}$ splits into four components, contained in $X \times X, X \times X^{\prime}, X^{\prime} \times X$ and $X^{\prime} \times X^{\prime}$ respectively. Of course, the desingularization $\tau: \widetilde{\Sigma} \rightarrow \Sigma$ splits as well. The first component gives $\Sigma_{1} \subset X \times X$, equipped with a desingularization $\widetilde{\Sigma}_{1} \rightarrow \Sigma_{1}$ induced by $\tau$.

On the other hand, we have by adjunction

$$
\left.\left(K_{\bar{X}}\right)\right|_{X}=\left.K_{Y}(\bar{X})\right|_{X}=\left.K_{Y}(X)\right|_{X} \otimes \vartheta_{X}\left(X^{\prime}\right)=K_{X}(D) .
$$

In particular, since $K_{\bar{X}}$ is trivial, $K_{X}(D)$ is trivial as well, and if $\eta_{\bar{X}}$ is a generator of $\mathrm{H}^{0}\left(\bar{X}, K_{\bar{X}}\right)$, then its restriction $\eta_{X}:=\left.\eta_{\bar{X}}\right|_{X}$ is a generator of $\mathrm{H}^{0}\left(X, K_{X}(D)\right)$. Since $X$ is smooth, $\eta_{X}$ is a meromorphic differential $n$-form, with polar divisor $D$. It is now clear from Theorem 4.7 that we have the equality of meromorphic $n$-forms

$$
m f_{1}^{*} \eta_{X}+m^{\prime} g_{1}^{*} \eta_{X}=0
$$

on the desingularization $\widetilde{\Sigma}_{1} \rightarrow \Sigma_{1}$. By Remark 4.5, this implies that $\Sigma_{1}$ is a log-K-autocorrespondence of $(X, D)$.

Proof of Theorem 4.7. - We consider a desingularization $\tau^{\prime}: \widetilde{\Sigma}^{\prime} \rightarrow \Sigma^{\prime}$, with maps $\widetilde{f}: \widetilde{\Sigma}^{\prime} \rightarrow \bar{X}$ and $\widetilde{g}: \widetilde{\Sigma}^{\prime} \rightarrow \bar{X}$. It is enough to show (20) on $\widetilde{\Sigma}^{\prime}$, that is

$$
m \tilde{f}^{*} \eta_{\bar{X}}=-m^{\prime} \tilde{g}^{*} \eta_{\bar{X}}
$$


The reason for this is that $\left(\Sigma, \mathrm{pr}_{1}, \mathrm{pr}_{2}\right)$ is the Stein factorization of $\left(\Sigma^{\prime}, f^{\prime}, g^{\prime}\right)$ (and also of $\left.\left(\widetilde{\Sigma}^{\prime}, \widetilde{f}, \widetilde{g}\right)\right)$. In fact, since (21) is an equality of meromorphic differential forms, it is enough to prove it locally, and on a dense open subset of $\tilde{\Sigma}^{\prime}$.

We construct three 0 -correspondences $\Gamma_{x, y}, \Gamma_{z}$ and $\Gamma_{C}$ between $\Sigma^{\prime}$ and $\bar{X}$. Each of them is defined by its fiber over a generic point $\sigma=(x, y, C) \in \Sigma^{\prime}$. We describe these generic fibers as subschemes of $\bar{X}$.

-The fiber of $\Gamma_{x, y}$ over $\sigma$ is the 0 -cycle $m x+m^{\prime} y$.

-The fiber of $\Gamma_{z}$ over $\sigma$ is the 0 -cycle $C \cdot X-m x-m^{\prime} y$. For $\sigma$ generic, this is the part of the intersection $C \cap \bar{X}$ that lies on the hypersurface $W \subset \bar{X}$.

-The fiber of $\Gamma_{C}$ over $\sigma$ is the 0 -cycle $C \cap \bar{X} . \Gamma_{C}$ can also be defined globally as $\mathscr{C} \cap\left(\Sigma^{\prime} \times \bar{X}\right) \subset \Sigma^{\prime} \times Y$, where $\mathscr{C}$ is the universal curve over $\Sigma^{\prime}$.

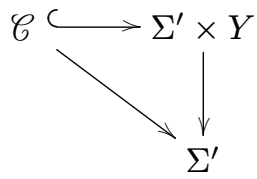

Now, following an idea of Mumford ([18]), we define the pull-back of $\eta_{\bar{X}}$ by a 0-correspondence $\Gamma \subset \Sigma^{\prime} \times \bar{X}$. In fact, since it is enough to prove (21) locally and on a dense open subset of $\widetilde{\Sigma}^{\prime}$, we only need to define the pull-back of the restriction of $\eta_{\bar{X}}$ to a dense open subset of $\bar{X}$. We thus define a smooth, dense open subset $\Sigma^{\circ} \subset \Sigma^{\prime}$ in the following way (since it is smooth, $\Sigma^{\circ}$ can also be seen as a dense open subset of $\left.\widetilde{\Sigma}^{\prime}\right)$. We let $\operatorname{pr}_{12}$ be the projection $\bar{X} \times \bar{X} \times\left|C_{0}\right| \rightarrow \bar{X} \times \bar{X}$. Then $\Sigma^{\circ}$ is the subset of $\Sigma^{\prime} \cap \operatorname{pr}_{12}^{-1}(\operatorname{sm}(\bar{X}) \times \operatorname{sm}(\bar{X}))$, above which the projection $\Gamma_{C} \rightarrow \Sigma^{\prime}$ is finite. By restricting $\Sigma^{\circ}$, one can assume that it is smooth, and that it is a self-0-correspondence of a smooth, dense open subset $X^{\circ} \subset \bar{X}$. We call $\Gamma^{\circ}$ the restriction of $\Gamma$ to $\Sigma^{\circ} \times X^{\circ}$, and $\varphi$ and $\psi$ the two projections of $\Gamma^{\circ}$ on $\Sigma^{\circ}$ and $X^{\circ}$ respectively. The key point here is the fact that $\varphi$ is proper. Of course, one can also assume $\Gamma^{\circ}$ to be smooth.

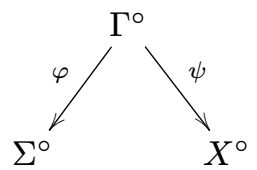

We now define the pull-back by $\Gamma^{\circ}$ of the restriction $\eta^{\circ}:=\left.\eta_{\bar{X}}\right|_{X^{\circ}}$. Since $X^{\circ} \subset \operatorname{sm}(\bar{X}), \eta^{\circ}$ is a holomorphic differential $n$-form on $X^{\circ}$. Its pull-back $\psi^{*} \eta^{\circ}$ is then a holomorphic $n$-form on $\Gamma^{\circ}$. Eventually, we let

$$
\left(\Gamma^{\circ}\right)^{*} \eta^{\circ}:=\varphi_{*} \psi^{*} \eta^{\circ},
$$

where $\varphi_{*}$ is the trace map relative to $\varphi .\left(\Gamma^{\circ}\right)^{*} \eta^{\circ}$ is a holomorphic $n$-form on $\Sigma^{\circ}$. It extends to a meromorphic $n$-form on the smooth variety $\widetilde{\Sigma}^{\prime}$; we call it $\Gamma^{*} \eta_{\bar{X}}$.

Tоме $138-2010-\mathrm{N}^{\mathrm{O}} 4$ 
The definition of the trace map $\varphi_{*}$ is classical. It goes as follows. Let $\omega$ be a holomorphic $n$-form on $\Gamma^{\circ}$. If $U \subset \Sigma^{\circ}$ is an open subset above which $\varphi$ is not ramified, then $\varphi^{-1} U$ is the disjoint union of $d$ open subsets $U_{1}, \ldots, U_{d} \subset \Gamma^{\circ}$, which are all isomorphic to $U$. On $U, \varphi_{*} \omega$ is defined as the sum

$$
\left.\omega\right|_{U_{1}}+\cdots+\left.\omega\right|_{U_{d}} \text {. }
$$

Then all these local definitions glue together to give a well defined holomorphic $n$-form $\varphi_{*} \omega$ on $\Sigma^{\circ}$, even if $\varphi$ ramifies on $\Gamma^{\circ}$.

We now have all the definitions we need to end the proof. The equality (21) is a simple consequence of the following proposition.

Proposition 4.9. - The holomorphic n-form $\left(\Gamma_{x, y}^{\circ}\right)^{*} \eta^{\circ}$ on $\Sigma^{\circ}$ vanishes.

To see why this implies (21), we let $\Gamma_{f^{\prime}}^{\circ} \subset \Sigma^{\circ} \times X^{\circ}$ and $\Gamma_{g^{\prime}}^{\circ} \subset \Sigma^{\circ} \times X^{\circ}$ denote the restrictions to $\Sigma^{\circ} \times X^{\circ}$ of the graphs of $f^{\prime}: \Sigma^{\prime} \rightarrow \bar{X}$ and $g^{\prime}: \Sigma^{\prime} \rightarrow \bar{X}$ respectively. By construction, we have the equality of $n$-cycles in $\Sigma^{\circ} \times X^{\circ}$

$$
\Gamma_{x, y}^{\circ}=m \Gamma_{f^{\prime}}^{\circ}+m^{\prime} \Gamma_{g^{\prime}}^{\circ}
$$

This implies that $\left(\Gamma_{x, y}^{\circ}\right)^{*} \eta^{\circ}=m f^{\prime *} \eta^{\circ}+m^{\prime} g^{*} \eta^{\circ}$. So Proposition 4.9 yields the equality (21) by continuity.

Proof of Proposition 4.9. - By construction, we have $\Gamma_{x, y}+\Gamma_{z}=\Gamma_{C}$ as $n$ cycles in $\Sigma^{\prime} \times \bar{X}$, and therefore $\Gamma_{x, y}^{\circ}+\Gamma_{z}^{\circ}=\Gamma_{C}^{\circ}$ in $\Sigma^{\circ} \times X^{\circ}$. The vanishing of proposition 4.9 is a consequence of the two vanishings $\left(\Gamma_{z}^{\circ}\right)^{*} \eta^{\circ}=\left(\Gamma_{C}^{\circ}\right)^{*} \eta^{\circ}=0$ of holomorphic $n$-forms on $\Sigma^{\circ}$.

The first one is given by the fact that $\Gamma_{z}$ is contained in $\Sigma^{\prime} \times W \subset \Sigma^{\prime} \times X$. Let $\nu: \widetilde{W} \rightarrow W^{\circ} \subset X^{\circ}$ be a desingularization of $W^{\circ}:=W \cap X^{\circ}$, and consider the following 0 -correspondence obtained by base change.

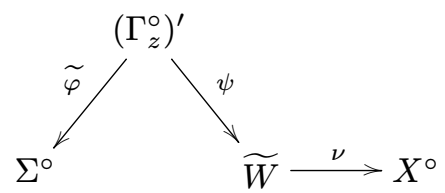

One has $\left(\Gamma_{z}^{\circ}\right)^{*} \eta^{\circ}=\left(\Gamma_{z}^{\circ}\right)^{\prime *} \nu^{*} \eta^{\circ}=0$, because $\operatorname{dim} W<n$ and therefore $\nu^{*} \eta^{\circ}=0$.

As for the second one, it is a consequence of proposition 4.10 below. This proposition says that we have an equality of meromorphic forms on $\widetilde{\Sigma}^{\prime}$

$$
\Gamma_{C}^{*} \eta_{\bar{X}}=\mathscr{C}^{*} l_{*} \eta_{\bar{X}}
$$

Here, $l_{*}: \mathrm{H}^{0}\left(\bar{X}, K_{\bar{X}}\right) \rightarrow \mathrm{H}^{1}\left(Y, K_{Y}\right)$ is a push-forward map induced by the inclusion $l: \bar{X} \subset Y$, and $\mathscr{C}^{*}: \mathrm{H}^{1}\left(Y, K_{Y}\right) \rightarrow \mathrm{H}^{0}\left(\widetilde{\Sigma}^{\prime}, K_{\widetilde{\Sigma}^{\prime}}\right)$ is given by the correspondence between smooth varieties $\widetilde{\mathscr{C}} \subset \widetilde{\Sigma}^{\prime} \times Y$, obtained by base change from the universal family $\mathscr{C} \subset \Sigma^{\prime} \times Y$. Now, since $Y$ is rationally connected, 
we have $\mathrm{H}^{1}\left(Y, K_{Y}\right)=0$. We deduce from this that $l_{*} \eta_{X}=0$, and therefore that $\Gamma_{C}^{*} \eta_{\bar{X}}=0$. A fortiori, we have $\left(\Gamma_{C}^{\circ}\right)^{*} \eta^{\circ}=0$ by restriction to $\Sigma^{\circ}$.

Let us now define precisely the various maps involved in formula (22). Let $l$ be the inclusion $\bar{X} \subset Y$. We define a push-forward morphism $l_{*}$, as the boundary morphism $\mathrm{H}^{0}\left(\bar{X}, K_{\bar{X}}\right) \rightarrow \mathrm{H}^{1}\left(Y, K_{Y}\right)$ of the long exact sequence associated to the Poincaré residue exact sequence

$$
0 \rightarrow K_{Y} \rightarrow K_{Y}(\bar{X}) \rightarrow K_{\bar{X}} \rightarrow 0 .
$$

On the other hand, we consider $\mathscr{C} \subset \Sigma^{\prime} \times Y$, which is the universal curve relative to the parametrizing variety $\Sigma^{\prime}$. We call $\Phi$ and $\Psi$ the two projections on $\Sigma^{\prime}$ and $Y$ respectively. By base change, $\mathscr{C}$ gives a correspondence in $\widetilde{\Sigma}^{\prime} \times$ $Y$. Desingularizing this new correspondence, we obtain the following diagram, where $\widetilde{\mathscr{C}}$ is smooth, as well as $\widetilde{\Sigma}^{\prime}$ and $Y$.

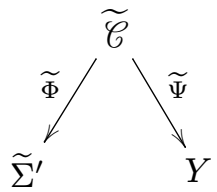

This yields a Mumford pull-back $\mathscr{C}^{*}: \mathrm{H}^{1}\left(Y, K_{Y}\right) \rightarrow \mathrm{H}^{0}\left(\widetilde{\Sigma}^{\prime}, K_{\widetilde{\Sigma}^{\prime}}\right)$, defined as the composition

$$
\mathrm{H}^{1}\left(Y, K_{Y}\right) \stackrel{\widetilde{\Psi}^{*}}{\longrightarrow} \mathrm{H}^{1}\left(\widetilde{\mathscr{C}}, K_{\widetilde{\mathscr{C}}}\right) \stackrel{\left(\widetilde{\Phi}^{*}\right)^{T}}{\longrightarrow} \mathrm{H}^{0}\left(\widetilde{\Sigma}^{\prime}, K_{\widetilde{\Sigma}^{\prime}}\right),
$$

where the last morphism is obtained by Serre duality as the transpose of the pull-back map $\Phi^{*}: \mathrm{H}^{n}\left(\widetilde{\Sigma}^{\prime}, \theta_{\widetilde{\Sigma}^{\prime}}\right) \rightarrow \mathrm{H}^{n}\left(\widetilde{\mathscr{C}}, \vartheta_{\widetilde{\mathscr{C}}}\right)$.

Proposition 4.10. - We have $\Gamma_{C}^{*} \eta_{\bar{X}}=\mathscr{C}^{*} l_{*} \eta_{\bar{X}}$, as an equality of meromorphic forms on $\widetilde{\Sigma}^{\prime}$.

Again, this is an equality of meromorphic forms, so we can prove it locally in the dense open subset $\Sigma^{\circ} \subset \widetilde{\Sigma}^{\prime}$, where it is an equality of holomorphic differential forms.

Now the situation is the following. $\mathscr{C}$ is a family of curves over $\Sigma^{\prime}$. These curves are embedded in $Y$. $\Gamma_{C}$ is cut out on $\mathscr{C} \subset \Sigma^{\prime} \times Y$ by $\Sigma^{\prime} \times \bar{X}$, and since $\bar{X} \subset Y$ is a divisor, $\Gamma_{C}$ can be seen as a family of divisors on the curves of the family $\mathscr{C}$. So basically, Proposition 4.10 is just a generalization of the following result on curves, which is an application of the residue theorem.

LEMma 4.11. - Let $C$ be a smooth curve, and $j: Z \subset C$ be an effective divisor. We have a push-forward morphism $j_{*}: \mathrm{H}^{0}\left(Z, K_{Z}\right) \rightarrow \mathrm{H}^{1}\left(C, K_{C}\right)$, 
taken from the long exact sequence associated to the Poincaré residue exact sequence

$$
0 \rightarrow K_{C} \rightarrow K_{C}(Z) \rightarrow K_{Z} \rightarrow 0 .
$$

The following diagram is commutative.

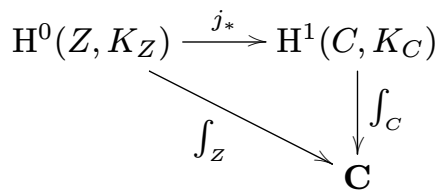

Proof. - We first study the case when $Z$ is a single point $x \in C$ with multiplicity $\mu$. It will then easily extend to the general case, as will be seen from the proof. We begin by defining a $C^{\infty}$ function

$$
\rho: z \in \mathbf{D} \mapsto \exp \left(\frac{|z|^{2}}{|z|^{2}-1}\right) \in \mathbf{R} .
$$

It extends by 0 on the complementary of the unit disk in $\mathbf{C}$ to a $C^{\infty}$ function $\mathbf{C} \rightarrow \mathbf{R}$. It also satisfies $\rho(0)=1$.

We then consider a neighbourhood $U \subset C$ of $x \in C$, equipped with a holomorphic coordinate $z$ centered at $x$, and such that $U \cong \mathbf{D}$ via $z$, and $Z \subset C$ is given by the equation $z^{\mu}=0$. Let $\omega_{Z} \in \mathrm{H}^{0}\left(Z, K_{Z}\right)$. It writes

$$
\frac{a_{-\mu+1}+a_{-\mu+2} z+\cdots+a_{0} z^{\mu-1}}{z^{\mu-1}} \in \mathbf{C}[z] /\left(z^{\mu}\right) \cdot \frac{1}{z^{\mu-1}},
$$

and we have $\int_{Z} \omega_{Z}=a_{0}$. The differential form

$$
\frac{\rho\left(z^{\mu}\right)}{2 \pi i}\left(a_{-\mu+1}+a_{-\mu+2} z+\cdots+a_{0} z^{\mu-1}\right) \frac{d z}{z^{\mu}}
$$

is sent to $\omega_{Z}$ by the residue map. Its $\bar{\partial}$-differential

$$
\frac{1}{2 \pi i} \frac{-\mu\left|z^{\mu-1}\right|^{2}}{\left(\left|z^{\mu}\right|^{2}-1\right)^{2}} \exp \left(\frac{\left|z^{\mu}\right|^{2}}{\left|z^{\mu}\right|^{2}-1}\right)\left(\frac{a_{-\mu+1}}{z^{\mu-1}}+\cdots+a_{0}\right) d \bar{z} \wedge d z
$$

thus represents $j_{*} \omega_{Z}$. Note that in (24), the pole $z^{\mu-1}$ is eliminated by the $\left|z^{\mu-1}\right|^{2}$ from the numerator. The differential form $j_{*} \omega_{Z}$ is therefore a $C^{\infty}$ section of $\Omega_{C}^{1,1}$. It is supported on $U \subset C$. The integral of this form on $\mathbf{D}$ is

$$
\begin{array}{r}
\frac{1}{2 \pi i} \int_{\mathbf{D}} \frac{-\mu\left(r^{\mu-1}\right)^{2}}{\left(r^{2 \mu}-1\right)^{2}} \exp \left(\frac{r^{2 \mu}}{r^{2 \mu}-1}\right)\left(\frac{a_{-\mu+1} e^{-i(\mu-1) \theta}}{r^{\mu-1}}+\cdots+a_{0}\right)(2 i r d r \wedge d \theta) \\
=a_{0} \int_{0 \leqslant r \leqslant 1} \frac{-2 r^{\mu}}{\left(r^{2 \mu}-1\right)^{2}} \exp \left(\frac{r^{2 \mu}}{r^{2 \mu}-1}\right)\left(\mu r^{\mu-1} d r\right)=a_{0} .
\end{array}
$$

This proves Lemma 4.11. 
The next step of the proof of Proposition 4.10 is to extend Lemma 4.11 to the family of curves $\mathscr{C}$. In fact, we have seen that it is enough to consider the smaller family $\mathscr{C}^{\circ} \rightarrow \Sigma^{\circ}$.

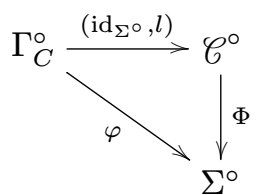

We recall that $\varphi$ and $\psi$ are the natural morphisms from $\Gamma_{C}$ to $\Sigma^{\prime}$ and $X$ respectively, that $\Phi$ and $\Psi$ are the natural morphisms from $\mathscr{C}$ to $\Sigma^{\prime}$ and $Y$, and that $l$ is the inclusion $\bar{X} \subset Y$. The following lemma is proved by using Lemma 4.11, and the fact that the push-forward map $\Phi_{*}$ is given locally above $\Sigma^{\circ}$ by the integration along the fibers of $\Phi$.

Lemma 4.12. - The following diagram is commutative.

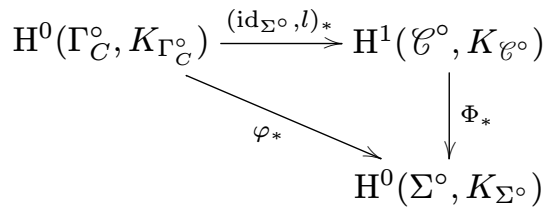

To be more precise, the vertical arrows of the preceding diagram are given by $\varphi_{*}^{\circ}$ and $\Phi_{*}^{\circ}$ respectively, where $\varphi^{\circ}:=\left.\varphi\right|_{\Gamma_{C}^{\circ}}$ and $\Phi^{\circ}:=\left.\Phi\right|_{\mathscr{C}^{\circ}}$.

Proof. - Again, we want to show an equality of holomorphic differential forms on $\Sigma^{\circ}$. This can be done locally. We thus choose an open subset $V \subset \Sigma^{\circ}$ over which the map $\Gamma_{C}^{\circ} \rightarrow \Sigma^{\circ}$ is étale (or rather the map $\left(\Gamma_{C}^{\circ}\right)_{r e d} \rightarrow \Sigma^{\circ}$ is étale). Then $\varphi^{-1}(V)$ is a disjoint union of open subsets $V_{j} \subset \Gamma_{C}^{\circ}$, such that for every $j$ one has $\left(V_{j}\right)_{\text {red }} \cong V$.

One can assume that $V$ is equipped with a holomorphic system of coordinates $v=\left(v_{1}, \ldots, v_{n}\right)$, and that there exists a neighbourhood $U_{j}$ of every $V_{j} \subset$ $\Phi^{-1}(V)$ equipped with a holomorphic system of coordinates $\left(v, t_{j}\right)$, such that $\Phi$ is simply given locally by $\left(v, t_{j}\right) \mapsto v$, and that $V_{j}$ is given by the local equation $t_{j}^{\mu_{j}}=0\left(\mu_{j}\right.$ is equal to $1, m$ or $\left.m^{\prime}\right)$. The coordinates $t_{j}$ give local parameters of the curves $C_{\sigma}(\sigma \in V)$ at their points of intersection with $\bar{X}$.

Let $\eta \in \mathrm{H}^{0}\left(\Gamma_{C}^{\circ}, K_{\Gamma_{C}^{\circ}}\right)$. Its restriction to $\varphi^{-1}(V)$ is a collection of meromorphic forms

$$
\eta_{j}=\left(a_{j,-\mu_{j}+1}(v)+a_{j,-\mu_{j}+2}(v) t_{j}+\cdots+a_{j, 0}(v) t_{j}^{\mu_{j}-1}\right) \frac{d v_{1} \wedge \cdots \wedge d v_{n}}{t_{j}^{\mu_{j}-1}}
$$

TOME $138-2010-\mathrm{N}^{\mathrm{O}} 4$ 
on the open subsets $V_{j}$, exactly as in (23). The map $\varphi_{*}$ is locally defined as the trace map, so $\varphi_{*} \eta$ is given in $V$ by

$$
\varphi_{*} \eta=\left(\sum_{j} a_{j, 0}(v)\right) d v_{1} \wedge \cdots \wedge d v_{n} .
$$

On the other hand, (id, $l)_{*} \eta$ is given in $\Phi^{-1}(V)$ by a $\bar{\partial}$-closed, $C^{\infty}$ differential form of type $(n+1,1)$, which vanishes outside from the neighbourhood $\bigcup_{j} U_{j}$ of $\Gamma_{C}^{\circ} \subset \mathscr{C}^{\circ}$. In the neighbourhood $U_{j}$ of $V_{j} \subset \Phi^{-1}(V)$, it is given by the $\bar{\partial}$-differential of

$\frac{\rho\left(t_{j}^{\mu_{j}}\right)}{2 \pi i}\left(a_{j,-\mu_{j}+1}(v)+a_{j,-\mu_{j}+2}(v) t_{j}+\cdots+a_{j, 0}(v) t_{j}^{\mu_{j}-1}\right) \frac{d t_{j}}{t_{j}^{\mu_{j}}} \wedge d v_{1} \wedge \cdots \wedge d v_{n}$, exactly as in the proof of Lemma 4.11. Eventually, $\Phi_{*}$ is given by the integration along the fibers of $\Phi$. To compute this, we let $\zeta$ be a holomorphic $n$-vector field over $V$ (i.e. a holomorphic section of $\bigwedge^{n} T_{\Sigma^{\circ}}$ over $V$ ), given in coordinates by

$$
h(v) \frac{\partial}{\partial v_{1}} \wedge \cdots \wedge \frac{\partial}{\partial v_{n}} .
$$

We lift it to a $C^{\infty} n$-vector field $\widetilde{\zeta}$ on $\Phi^{-1}(V)$. Using a partition of unity, we can assume that in every $U_{j}, \widetilde{\zeta}$ is simply given by the expression (25). At a point $\sigma \in V$ of coordinates $v$, the inner product $\left(\Phi_{*}(\mathrm{id}, l)_{*} \eta\right)(\zeta)_{\sigma}$ is by definition

$$
\int_{C_{\sigma}}\left((\mathrm{id}, l)_{*} \eta\right)(\widetilde{\zeta})
$$

where $C_{\sigma}$ is the fiber of $\Phi$ over $\sigma$. We let $Z_{\sigma}:=\left(\Gamma_{C}^{\circ} \cap C_{\sigma}\right)$, and denote by $j_{\sigma}$ the inclusion $Z_{\sigma} \subset C_{\sigma}$. The inner product $\eta(\widetilde{\zeta})_{Z_{\sigma}}:=\sum_{z \in \operatorname{Supp}\left(Z_{\sigma}\right)} \eta(\widetilde{\zeta})_{z}$ sits naturally in $\mathrm{H}^{0}\left(Z_{\sigma}, K_{Z_{\sigma}}\right)$. Now the $(1,1)$-form $\left((\mathrm{id}, l)_{*} \eta\right)(\widetilde{\zeta})$ restricted to $C_{\sigma}$ is precisely $\left(j_{\sigma}\right)_{*}\left(\eta(\widetilde{\zeta})_{Z_{\sigma}}\right)$, so by Lemma 4.11 , we have

$$
\left(\Phi_{*}(\mathrm{id}, l)_{*} \eta\right)(\zeta)_{\sigma}=\int_{C_{\sigma}}\left(j_{\sigma}\right)_{*}\left(\eta(\widetilde{\zeta})_{Z_{\sigma}}\right)=\int_{Z_{\sigma}} \eta(\widetilde{\zeta})_{Z_{\sigma}}=\left(\varphi_{*} \eta\right)(\zeta)_{\sigma}
$$

This shows the equality of holomorphic differential forms $\Phi_{*}(\mathrm{id}, l)_{*} \eta=\varphi_{*} \eta$ on $V$.

To conclude the proof of Proposition 4.10, it only remains to show the following commutativity result.

Lemma 4.13. - The following diagram is commutative.

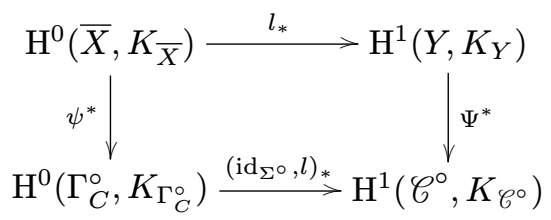


In fact, the vertical arrows of this diagram are rather $\left(\psi^{\circ}\right)^{*}$ and $\left(\Psi^{\circ}\right)^{*}$, where $\psi^{\circ}: \Gamma_{C}^{\circ} \rightarrow \bar{X}$ and $\Psi^{\circ}: \mathscr{C}^{\circ} \rightarrow Y$ are the restrictions of $\psi$ and $\Psi$ to $\Gamma_{C}^{\circ}$ and $\mathscr{C}^{\circ}$ respectively.

Proof. - It is clear from the definitions that $\psi^{\circ}=\left.\Psi^{\circ}\right|_{\Gamma_{C}^{\circ}}$. On the other hand, we have $\Psi^{*} \bar{X}=\Gamma_{C}^{\circ}$ as an equality of divisors on $\mathscr{C}^{\circ}$. This shows that we have a morphism of short exact sequences as follows.

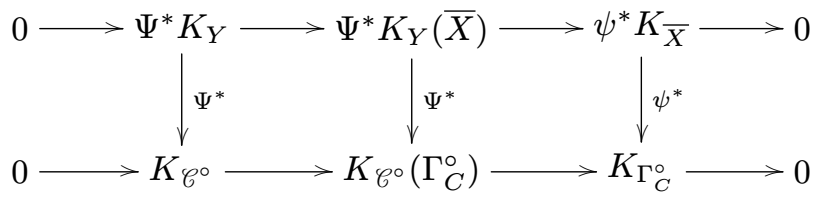

It yields a morphism between the associated long exact sequences in cohomology. In particular, the following diagram is commutative.

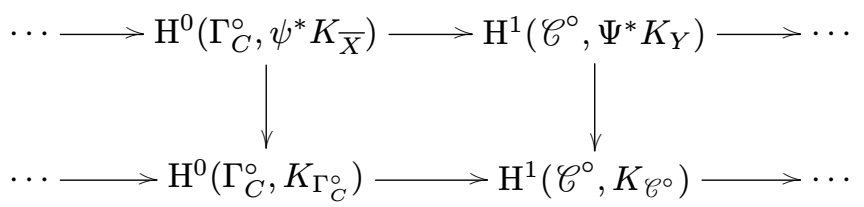

Up to the shrinking of $\Sigma^{\circ} \subset \Sigma$, we can assume that both $\psi^{\circ}$ and $\Psi^{\circ}$ are smooth, so we have $\mathrm{H}^{0}\left(\Gamma_{C}^{\circ}, \psi^{*} K_{\bar{X}}\right) \cong \mathrm{H}^{0}\left(\bar{X}, K_{\bar{X}}\right)$ and $\mathrm{H}^{1}\left(\mathscr{C}^{\circ}, \Psi^{*} K_{Y}\right) \cong \mathrm{H}^{1}\left(Y, K_{Y}\right)$, and the lemma is proved.

Eventually, Lemmas 4.12 and 4.13 give the commutativity of the left-hand side square and of the right-hand side triangle in the following diagram.

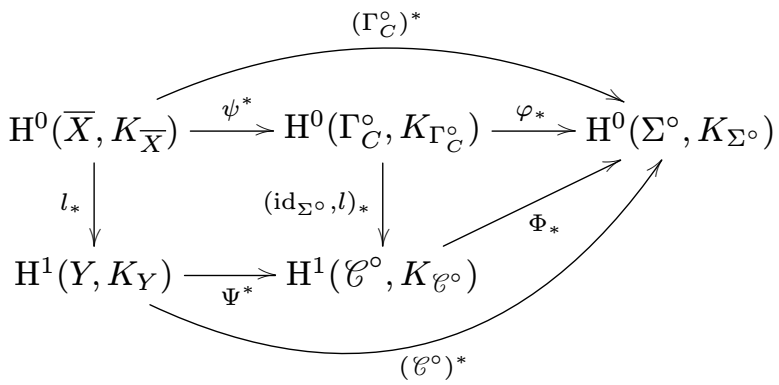

On the other hand, it follows from the definitions of $\left(\Gamma_{C}^{\circ}\right)^{*}$ and $\left(\mathscr{C}^{\circ}\right)^{*}$ that the bended arrows commute with the rest of the diagram. We thus have the equality of holomorphic forms on $\Sigma^{\circ}$

$$
\left(\Gamma_{C}^{\circ}\right)^{*} \eta^{\circ}=\left(\mathscr{C}^{\circ}\right)^{*} l_{*} \eta_{\bar{X}},
$$

TOME $138-2010-\mathrm{N}^{\mathrm{O}} 4$ 
which yields by continuity the desired equality of meromorphic differential forms on $\widetilde{\Sigma}^{\prime}$. This ends the proof of proposition 4.10. Theorem 4.7 is thus completely proved.

\section{BIBLIOGRAPHY}

[1] S. BLOCH - "On an argument of Mumford in the theory of algebraic cycles", in Journées de Géometrie Algébrique d'Angers, Juillet 1979/Algebraic Geometry, Angers, 1979, Sijthoff \& Noordhoff, 1980, p. 217-221.

[2] S. Bloch \& V. SRINivas - "Remarks on correspondences and algebraic cycles", Amer. J. Math. 105 (1983), p. 1235-1253.

[3] F. Campana - "Orbifolds, special varieties and classification theory", Ann. Inst. Fourier (Grenoble) $\mathbf{5 4}$ (2004), p. 499-630.

[4] J. Carlson \& P. A. Griffiths - "A defect relation for equidimensional holomorphic mappings between algebraic varieties", Ann. of Math. 95 (1972), p. 557-584.

[5] L. Clozel \& E. Ullmo - "Correspondances modulaires et mesures invariantes", J. reine angew. Math. 558 (2003), p. 47-83.

[6] J.-P. DEMAILLY - "Algebraic criteria for Kobayashi hyperbolic projective varieties and jet differentials", in Algebraic geometry-Santa Cruz 1995, Proc. Sympos. Pure Math., vol. 62, Amer. Math. Soc., 1997, p. 285-360.

[7] J.-P. Demailly, L. Lempert \& B. Shiffman - "Algebraic approximations of holomorphic maps from Stein domains to projective manifolds", Duke Math. J. 76 (1994), p. 333-363.

[8] H. M. FARKAS \& I. KRA - Riemann surfaces, second ed., Graduate Texts in Math., vol. 71, Springer, 1992.

[9] M. Green \& P. A. Griffiths - "Two applications of algebraic geometry to entire holomorphic mappings", in The Chern Symposium 1979 (Proc. Internat. Sympos., Berkeley, Calif., 1979), Springer, 1980, p. 41-74.

[10] P. A. Griffiths - "Holomorphic mapping into canonical algebraic varieties", Ann. of Math. 93 (1971), p. 439-458.

[11] _ Entire holomorphic mappings in one and several complex variables, Princeton Univ. Press, 1976, The fifth set of Hermann Weyl Lectures, given at the Institute for Advanced Study, Princeton, N. J., October and November 1974, Annals of Mathematics Studies, No. 85.

[12] R. KoBAYASHI - "Kähler-Einstein metric on an open algebraic manifold", Osaka J. Math. 21 (1984), p. 399-418.

[13] S. Kobayashi - "Intrinsic distances, measures and geometric function theory", Bull. Amer. Math. Soc. 82 (1976), p. 357-416. 
[14] S. Kobayashi \& T. OCHIAI - "Mappings into compact manifolds with negative first Chern class", J. Math. Soc. Japan 23 (1971), p. 137-148.

[15] _ "Meromorphic mappings onto compact complex spaces of general type", Invent. Math. 31 (1975), p. 7-16.

[16] J. Kollár, Y. Miyaoka \& S. Mori - "Rationally connected varieties", J. Algebraic Geom. 1 (1992), p. 429-448.

[17] S. LANG - "Hyperbolic and Diophantine analysis", Bull. Amer. Math. Soc. (N.S.) 14 (1986), p. 159-205.

[18] D. Mumford - "Rational equivalence of 0-cycles on surfaces", J. Math. Kyoto Univ. 9 (1968), p. 195-204.

[19] G. TIAN \& S. T. YAU - "Existence of Kähler-Einstein metrics on complete Kähler manifolds and their applications to algebraic geometry", in Mathematical aspects of string theory (San Diego, Calif., 1986), Adv. Ser. Math. Phys., vol. 1, World Sci. Publishing, 1987, p. 574-628.

[20] K. UENO - Classification theory of algebraic varieties and compact complex spaces, Lecture Notes in Math., vol. 439, Springer, 1975.

[21] E. Ullmo \& A. YAfaev - "Points rationnels des variétés de Shimura : un principe du "tout ou rien"", preprint, 2007.

[22] C. VoIsin - Théorie de Hodge et géométrie algébrique complexe, Cours Spécialisés, vol. 10, Soc. Math. France, 2002.

[23] _ "On some problems of Kobayashi and Lang; algebraic approaches", in Current developments in mathematics, 2003, Int. Press, Somerville, MA, 2003, p. 53-125.

[24] _ "Intrinsic pseudo-volume forms and $K$-correspondences", in The Fano Conference, Univ. Torino, Turin, 2004, p. 761-792.

[25] S. T. YAU - "Intrinsic measures of compact complex manifolds", Math. Ann. 212 (1975), p. 317-329. 